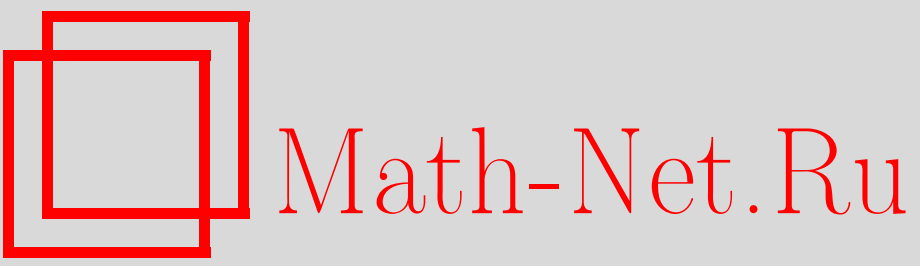

А. М. Райгородский, Проблемы Борсука и Грюнбаума для решетчатых многогранников, Изв. РАН. Сер. матем., 2005, том 69, выпуск 3, 81-108

DOI: https://doi.org/10.4213/im641

Использование Общероссийского математического портала Math-Net.Ru подразумевает, что вы прочитали и согласны с пользовательским соглашением

http://www.mathnet.ru/rus/agreement

Параметры загрузки:

IP : 107.22 .136 .117

26 апреля 2023 г., 11:39:04 
УДК $514.17+519.174$

\author{
А.М. Райгородский
}

\title{
Проблемы Борсука и Грюнбаума для решетчатых многогранников
}

\begin{abstract}
Изучаются две классические задачи комбинаторной геометрии: проблема Борсука о разбиении множеств на части меньшего диаметра и проблема Грюнбаума о покрытии множеств шарами. Получены новые нетривиальные верхние оценки минимального числа частей меньшего диаметра, на которые может быть разбит произвольный решетчатый многогранник, и минимального числа шаров того же диаметра, которыми всякий такой многогранник может быть покрыт.

Библиография: 37 наименований.
\end{abstract}

\section{§1. Введение}

Настоящая работа посвящена изучению двух классических проблем комбинаторной геометрии: проблемы Борсука и проблемы Грюнбаума. Первая проблема, сформулированная К. Борсуком в 1933 г. (см. [1]), состоит, по существу, в нахождении минимального числа $f(d)$ такого, что всякое ограниченное неодноточечное множество $\Omega \subset \mathbb{R}^{d}$ может быть разбито на $f(d)$ частей, имеющих диаметры, меньшие, чем диаметр $\Omega$. Проблема Грюнбаума (см. [2]-[5]), возникшая в 50-е годы прошлого века, сводится, в свою очередь, к определению наименьшей величины $g(d)$, обладающей тем свойством, что каждое множество $\Omega \subset \mathbb{R}^{d}, \operatorname{diam} \Omega<\infty$, покрывается $g(d)$ шарами того же диаметра. В действительности, существует принципиальное различие между ситуациями, когда рассматриваемые шары открыты и когда они замкнуты. Однако далее нас будут интересовать, главным образом, оценки величин $f(d)$ и $g(d)$, которые удается получить в предположении неограниченного роста размерности, а при $d \rightarrow \infty$ упомянутое различие практически исчезает и на искомые оценки влияния не оказывает. В результате мы можем изначально предполагать, например, что шары в проблеме Грюнбаума замкнуты.

Понятно, что проблемы, которые рассмотрены в статье, тесно связаны между собою. В частности, почти очевидно неравенство $f(d) \leqslant(d+1) g(d)$, вытекающее из того факта, что всякий шар в $\mathbb{R}^{d}$ легко может быть разрезан на $d+1$ часть меньшего диаметра (см., например, [6] или [7]). Обратное соотношение представляется уже гораздо более проблематичным, и мы увидим, что это не случайно: несмотря на явную близость задач соответствуюшие результаты в значительной мере отличаются друг от друга.

Работа выполнена при финансовой поддержке гранта Президента РФ № МK-3130.2004.1 и гранта ИНТАС №03-51-5070, а также гранта поддержки Ведущих научных школ НШ-136.2003.1. 
Перейдем теперь к краткому изложению истории вопроса. Долгое время (шестьдесят лет) сушествовала гипотеза о том, что в проблеме Борсука $f(d)=d+1$. В самом деле, оценка $f(d) \geqslant d+1$ следует из рассмотрения множества вершин правильного $d$-мерного симплекса (принцип Дирихле). В некоторых частных случаях (например, в случае гладкого тела) выполняется и противоположное неравенство (т.е., скажем, всякое гладкое тело допускает разбиение на $d+1$ часть меньшего диаметра [8]). Гипотеза была опровергнута лишш в 1993 г. Дж. Каном и Г. Калаи (см. [9]), причем авторы контрпримера заменили гипотетическую линейную функцию в оценке субэкспоненциальной:

$$
f(d) \geqslant(1.203 \ldots+o(1))^{\sqrt{d}} .
$$

В 1999 г. автор настоящей работы улучшил упомянутый результат (см. [10]), и на данньй момент самой сильной из известных остается оценка

$$
f(d) \geqslant\left(\frac{2}{\sqrt{3}}+o(1)\right)^{\sqrt{2} \sqrt{d}}=(1.2255 \ldots+o(1))^{\sqrt{d}} .
$$

Верхние оценки величины $f(d)$ были получены в следующей последовательности:

$$
f(d) \leqslant(\sqrt{2}+o(1))^{d}
$$

(К.А. Роджерсом в 1963 г. [11]),

$$
f(d) \leqslant\left(\sqrt{\frac{3}{2}}+o(1)\right)^{d}=(1.224 \ldots+o(1))^{d}
$$

(О. Шраммом в 1988 г. [12], Ж. Бургейном и Й. Линденштрауссом в 1991 г. [13]).

Заметим, во-первых, что сушествует еше несколько промежуточных неравенств, однако здесь мы их не приводим, рекомендуя читателю подробный обзор автора [14], во-вторых, что сохраняется значительный зазор между верхними и нижними оценками

$$
(1.2255 \ldots+o(1))^{\sqrt{d}} \leqslant f(d) \leqslant(1.224 \ldots+o(1))^{d}
$$

и что, безусловно, любое уменьшение этого зазора представляет большой интерес. Заметим, наконец, что оценки Роджерса и Бургейна-Линденштраусса получены как раз за счет неравенства $f(d) \leqslant(d+1) g(d)$, так что для $g(d)$ мы также не имеем пока верхних оценок, лучших, чем указанная экспонента. Тем не менее (и здесь возникает обешанное отличие), в проблеме Грюнбаума столь существенного зазора, как в проблеме Борсука, нет: еше в 1965 г. Л. Данцер показал, что $g(d) \geqslant(1.003)^{d}$ (см. [15]), а в 1991 г. Бургейн и Линденштраусс (см. [13]) усилили это неравенство, заменив его правую часть величиной $(1.0645)^{d}$.

Отметим, что все самые сильные нижние оценки величин $f(d)$ и $g(d)$ были получены с помощью многогранников, причем для оценки $f(d)$ использовались 
peшетчатые многогранники весьма специального вида: $(0,1)$-многогранники и $(-1,0,1)$-многогранники (кросс-политопы). В связи с этим естественно возникает вопрос: нельзя ли именно на классе решетчатых многогранников добиться уточнения не вполне удовлетворительных верхних оценок в задачах? Положительный ответ на этот вопрос тем более интересен, что, как уже упоминалось, мы не знаем примеров множеств, используя которые можно улучшить нижние оценки величин $f(d)$ и $g(d)$, полученные с помощью специальных многогранников. Изучению поставленного вопроса в последние годы было посвящено немало работ. Так, например, в серии статей [16]-[19] (см. также [20]) авторам удалось показать, что всякий $(0,1)$-многогранник размерности $d$ не более девяти можно разбить на $d+1$ часть меньшего диаметра. В асимптотическом случае аналогичные исследования производились преимущественно автором, которому удалось существенно усилить оценки Шрамма и Бургейна-Линденштраусса на достаточно широких классах $(0,1)$-многогранников, кросс-политопов и решетчатых многогранников общего вида. Для задачи Борсука такие оценки были получены в работах [21]-[24] (см., кроме того, [25]); для задачи Грюнбаума - в статьях [24] и [26]. Более детальное изучение вопроса приводит, однако, к дальнейшим продвижениям в решении проблем. В настоящей работе мы сформулируем и докажем все самые точные результаты, которые так или иначе удается установить для проблем Борсука и Грюнбаума в случае решетчатых многогранников. Мы отдельно рассмотрим ситуацию с $(0,1)$-многогранниками, отдельно обсудим кросс-политопы и общую ситуацию.

Прежде чем переходить к изложению необходимых дополнительных понятий и, собственно, к формулировкам, заметим, что некоторые частные результаты могут быть также найдены в работе [27].

\section{§2. Определения и обозначения}

Будем следовать тем определениям и обозначениям, которые были введены в краткой заметке автора [24]. Итак, рассмотрим евклидово пространство $\mathbb{R}^{d}$ и зафиксируем произвольные вещественные числа $a_{1}, \ldots, a_{t}$, а также произвольные натуральные числа $l_{a_{1}}, \ldots, l_{a_{t}}$, удовлетворяющие условию $l_{a_{1}}+\cdots+l_{a_{t}}=d$. Ясно, что, во всяком случае, $t \leqslant d$. Определим $\mathfrak{F}\left(l_{a_{1}}, \ldots, l_{a_{t}} ; k ; d\right), k \in \mathbb{R}$, как класс всех возможных совокупностей векторов $\mathcal{F}\left(l_{a_{1}}, \ldots, l_{a_{t}} ; k ; d\right)=\left\{\mathbf{v}_{1}, \ldots, \mathbf{v}_{s}\right\} \subset \mathbb{R}^{d}$, для которых выполнены следующие свойства:

(i) полагая $\mathbf{v}_{i}=\left(v_{i}^{1}, \ldots, v_{i}^{d}\right), i=1, \ldots, s$, для любых $i \in\{1, \ldots, s\}$ и $j \in$ $\{1, \ldots, t\}$ имеем card $\left\{\nu=1, \ldots, d: v_{i}^{\nu}=a_{j}\right\}=l_{a_{j}}$;

(ii) $\left(\operatorname{diam} \mathcal{F}\left(l_{a_{1}}, \ldots, l_{a_{t}} ; k ; d\right)\right)^{2}=k$.

Таким образом, $\mathfrak{F}\left(l_{a_{1}}, \ldots, l_{a_{t}} ; k ; d\right)$ - класс всех совокупностей $d$-мерных векторов, имеющих для любого $j \in\{1, \ldots, t\}$ в точности $l_{a_{j}}$ координат, равных величине $a_{j}$; кроме того, квадрат диаметра всякой такой совокупности совпадает c $k$. С одной стороны, корректность данного определения вытекает из соотношения $l_{a_{1}}+\cdots+l_{a_{t}}=d$. С другой стороны, понятно, что свойство (ii) накладывает дополнительные естественные ограничения на множества допустимых значений величин $l_{a_{1}}, \ldots, l_{a_{t}}, k$. Наконец, величина $s=\operatorname{card} \mathcal{F}\left(l_{a_{1}}, \ldots, l_{a_{t}} ; k ; d\right)$ заведомо на- 
ходится в пределах от единицы до $S$, где

$$
S=\left(\begin{array}{c}
d \\
l_{a_{1}}
\end{array}\right) \times\left(\begin{array}{c}
d-l_{a_{1}} \\
l_{a_{2}}
\end{array}\right) \times \cdots \times\left(\begin{array}{c}
d-l_{a_{1}}-\cdots-l_{a_{t-1}} \\
l_{a_{t}}
\end{array}\right) .
$$

В силу свойства (ii) упомянутая верхняя оценка может быть сушественно улучшена, но в дальнейшем такого рода улучшения нам не понадобятся, и в настояшей работе мы их не приводим.

На классе $\mathfrak{F}\left(l_{a_{1}}, \ldots, l_{a_{t}} ; k ; d\right)$ введем величины $f\left(l_{a_{1}}, \ldots, l_{a_{t}} ; k ; d\right)$ и $g\left(l_{a_{1}}, \ldots\right.$ $\left.\ldots, l_{a_{t}} ; k ; d\right)$, определяя первую из них как минимальное число частей меньшего диаметра, на которые может быть разбита каждая совокупность векторов из класса, а вторую - как наименьшее число шаров того же диаметра, которыми покрывается всякая такая совокупность. Ясно, что величина $f\left(l_{a_{1}}, \ldots, l_{a_{t}} ; k ; d\right)$ отвечает проблеме Борсука, а $g\left(l_{a_{1}}, \ldots, l_{a_{t}} ; k ; d\right)$ - проблеме Грюнбаума. Кроме того, нетрудно перейти от совокупностей векторов к многогранникам, заменяя эти совокупности своими выпуклыми оболочками и преобразуя, тем самым, класс $\mathfrak{F}\left(l_{a_{1}}, \ldots, l_{a_{t}} ; k ; d\right)$ в класс многогранников $\mathfrak{O}\left(l_{a_{1}}, \ldots, l_{a_{t}} ; k ; d\right)$. В частности, полагая $a_{1}=0, a_{2}=1$ или $a_{1}=0, a_{2}=1, a_{3}=-1$, получаем соответственно задачи для $(0,1)$-многогранников или кросс-политопов. Заметим, что класс $\mathfrak{O}$ в определенном смысле шире класса решетчатых многогранников (т.е. тел, являющихся выпукльми оболочками точек какой-либо решетки в $\left.\mathbb{R}^{d}\right)$.

В следующем параграфе мы укажем верхние оценки величин $f\left(l_{a_{1}}, \ldots, l_{a_{t}} ; k ; d\right)$ и $g\left(l_{a_{1}}, \ldots, l_{a_{t}} ; k ; d\right)$. Эти оценки во многих случаях окажутся сильнее как результатов Бургейна-Линденштраусса, так и некоторых из прежних результатов автора.

\section{§ 3. Формулировки результатов}

Для удобства изложения настояший параграф разделен на три части: в первой сформулируем различные результаты, полученные для $(0,1)$-многогранников, т. е. приведем верхние оценки величин $f\left(l_{0}, l_{1} ; k ; d\right)$ и $g\left(l_{0}, l_{1} ; k ; d\right)$; во второй обсудим аналогичные формулировки для кросс-политопов и отвечающих им числовых характеристик; в третьей рассмотрим общую ситуацию. Определим сначала величину $G(D, L, S)$ как вешественнозначную функцию трех вешественных переменных, получаемую из равенства

$$
G(D, L, S)=\max \left\{\frac{D}{L} \log \frac{S L}{D}, \frac{D}{L}\right\}+\frac{D}{L}+1 .
$$

Здесь переменные $D, L, S$ удовлетворяют естественным ограничениям, при которых правая часть выражения (2) корректно определена; через log обозначен натуральный логарифм.

Далее мы будем пользоваться формулой (2), в зависимости от контекста надлежащим образом задавая значения величин $D, L, S$.

3.1. Случай $(0,1)$-многогранников. Изучим здесь проблему Борсука и отдельно проблему Грюнбаума.

Проблема Борсука. Проблема Борсука для $(0,1)$-многогранников была детально изучена автором в работах [21]-[23], так что здесь мы лишш выпишем самые сильные из полученных результатов. Имеют место следующие три теоремы. 
Tеорема 1. Выполнена оценка

$$
f\left(l_{0}, l_{1} ; k ; d\right) \leqslant d \min \left\{\left(\begin{array}{c}
l_{1} \\
l_{1}-\frac{k}{2}
\end{array}\right),\left(\begin{array}{c}
l_{0} \\
\frac{k}{2}
\end{array}\right)\right\} .
$$

Tеорема 2. Пусть произвольные числа $x \in \mathbb{N} \cup\{0\}$ и $y \in \mathbb{N} \cup\{0\}$ таковы, что $x \leqslant l_{1}, \quad y \leqslant l_{0}$ u $2\left(\min \{x, y\}+\min \left\{l_{1}-x, l_{0}-y\right\}\right)<k$. Обозначим через $D_{1}=D_{1}(x, y)$ величину $\left(\begin{array}{c}d \\ x+y\end{array}\right)$, через $L_{1}=L_{1}(x, y)$ величину $\left(\begin{array}{c}l_{1} \\ x\end{array}\right)\left(\begin{array}{c}l_{0} \\ y\end{array}\right)$, а через $S_{1}$ величину $\left(\begin{array}{l}d \\ l_{1}\end{array}\right)$. Тогда

$$
f\left(l_{0}, l_{1} ; k ; d\right) \leqslant \min _{x, y} G\left(D_{1}(x, y), L_{1}(x, y), S_{1}\right) .
$$

Здесь минимум берется по всем допустимым значениям параметров х и у.

Последняя теорема дает исчерпьвающий ответ на вопрос, при каких значениях параметров $x, y$ достигается минимум в оценке (4).

Теорема 3. Имеется альтернатива:

а) если $d \geqslant\left(l_{1}-t+1\right)(t+1)$, где $t=l_{1}-\frac{k}{2}+1$, то минимум в неравенстве (4) достигается при $x=t, y=0$;

b) если $d<\left(l_{1}-t+1\right)(t+1)$, то минимум в неравенстве (4) достигается при $x=t+r, \quad y=r$, где натуральное число $r$ однозначно находится из соотношений $t+r \leqslant l_{1}, \quad r \leqslant l_{0} u$

$$
\left(l_{1}-t+1\right)\left(2+\frac{t-1}{r+1}\right) \leqslant d \leqslant\left(l_{1}-t+1\right)\left(2+\frac{t-1}{r}\right) .
$$

ЗАмЕчАнИЕ 1. В теоремах 1-3 мы неявно предполагали, что $\frac{k}{2}$ - целое число. Априори это, казалось бы, неверно. Однако это именно так, поскольку квадрат расстояния между любыми двумя $(0,1)$-векторами с фиксированным числом координат каждого типа заведомо четен, а значит, четен и квадрат диаметра произвольной совокупности таких векторов.

Проблема Грюнбаума. Некоторые из результатов, которые мы выпишем ниже, в неявном виде содержались в работах автора [24] и [26]. Здесь мы изложим все оценки максимально подробно, это поможет лучше понять общую схему построения неравенств.

Чтобы сформулировать дальнейшие теоремы, нам потребуются дополнительные параметры: вектор $\mathfrak{g}=\left(g_{1}, g_{1 / 2}, g_{0}\right)$ и матрица

$$
\mathfrak{G}=\left(\begin{array}{cc}
g_{1}^{0} & g_{1}^{1} \\
g_{1 / 2}^{0} & g_{1 / 2}^{1} \\
g_{0}^{0} & g_{0}^{1}
\end{array}\right),
$$

элементы которых удовлетворяют следуюшим условиям: 
1) $g_{i}$ и $g_{i}^{j}$ - неотрицательные целые числа при всех значениях индексов $i \in$ $\{1,1 / 2,0\}, j \in\{0,1\}$

2) для каждого $i \in\{1,1 / 2,0\}$ выполнено равенство $g_{i}^{0}+g_{i}^{1}=g_{i}$;

3) для каждого $j \in\{0,1\}$ выполнено равенство $g_{1}^{j}+g_{1 / 2}^{j}+g_{0}^{j}=l_{j}$;

4) $g_{1}+g_{1 / 2}+g_{0}=\sum_{i \in\{1,1 / 2,0\}} \sum_{j \in\{0,1\}} g_{i}^{j}=d$;

5) $\sum_{i \in\{1,1 / 2,0\}} \sum_{j \in\{0,1\}}(i-j)^{2} g_{i}^{j} \leqslant \frac{k}{4}$.

Заметим, что верхние индексы везде отвечают допустимым значениям координат векторов в рассматриваемой задаче. Нижние индексы можно интерпретировать как полусуммы таких значений. Положим, далее, $D_{2}=P\left(g_{1}, g_{1 / 2}, g_{0}\right)$, где $P\left(u_{1}, \ldots, u_{r}\right)=\frac{\left(u_{1}+\cdots+u_{r}\right) !}{u_{1} ! \ldots u_{r} !}-$ полиномиальньй коэффициент. Положим также $L_{2}=P\left(g_{1}^{0}, g_{1 / 2}^{0}, g_{0}^{0}\right) \times P\left(g_{1}^{1}, g_{1 / 2}^{1}, g_{0}^{1}\right)$ и $S_{2}=S_{1}=\left(\begin{array}{c}d \\ l_{1}\end{array}\right)$. Имеет место

Tеорема 4. Выполнена оценка

$$
g\left(l_{0}, l_{1} ; k ; d\right) \leqslant \min _{\mathfrak{g}, \mathfrak{G}} G\left(D_{2}, L_{2}, S_{2}\right)
$$

Возможность и целесообразность точного определения параметров $g_{i}$ и $g_{i}^{j}$, на которых достигается минимум в оценке (6), мы обсудим после доказательства наших результатов, когда прояснится геометрический смысл этих параметров. Поэтому аналог теоремы 3 мы не формулируем.

Теперь приведем еще одно утверждение, во многих случаях улучшающее теорему 4. Положим $d^{\prime}=l_{0}+\frac{k}{2}, d^{\prime \prime}=l_{1}+\frac{k}{2}, l_{1}^{\prime}=\frac{k}{2}, l_{1}^{\prime \prime}=l_{1}, l_{0}^{\prime}=l_{0}, l_{0}^{\prime \prime}=\frac{k}{2}$. Для каждого из наборов $d^{\prime}, l_{0}^{\prime}, l_{1}^{\prime}$ и $d^{\prime \prime}, l_{0}^{\prime \prime}, l_{1}^{\prime \prime}$ зададим произвольным образом параметры $\mathfrak{g}^{\prime}, \mathfrak{G}^{\prime}$ и $\mathfrak{g}^{\prime \prime}, \mathfrak{G}^{\prime \prime}$, формально обладаюшие теми же пятью свойствами, что и $\mathfrak{g}, \mathfrak{G}$. Пусть, наконец, $D_{2}^{\prime}, L_{2}^{\prime}, S_{2}^{\prime}$ и $D_{2}^{\prime \prime}, L_{2}^{\prime \prime}, S_{2}^{\prime \prime}$ - полные аналоги величин $D_{2}$, $L_{2}, S_{2}$ с точностью до замены параметров без штрихов на параметры со штрихами. Тогда верна

Теорема 5. Выполнена оценка

$$
\begin{aligned}
& g\left(l_{0}, l_{1} ; k ; d\right) \\
& \quad \leqslant \min \left\{\left(\begin{array}{c}
l_{1} \\
l_{1}-\frac{k}{2}
\end{array}\right) \min _{\mathfrak{g}^{\prime}, \mathfrak{G}^{\prime}} G\left(D_{2}^{\prime}, L_{2}^{\prime}, S_{2}^{\prime}\right),\left(\begin{array}{c}
l_{0} \\
\frac{k}{2}
\end{array}\right) \min _{\mathfrak{g}^{\prime \prime}, \mathfrak{G}^{\prime \prime}} G\left(D_{2}^{\prime \prime}, L_{2}^{\prime \prime}, S_{2}^{\prime \prime}\right)\right\} .
\end{aligned}
$$

Отметим, что прослеживается некоторая аналогия теоремы 5 с теоремой 1.

3.2. Случай $(-1,0,1)$-многогранников (кросс-политопов). Как и в предыдущем пункте, мы рассмотрим отдельно проблему Борсука и отдельно проблему Грюнбаума.

Проблема Борсука. Для того чтобы сформулировать три следуюшие теоремы, введем некоторые дополнительные параметры. Пусть вектор $\mathfrak{m}=\left(m_{1}, m_{2}, m_{3}\right)$ и матрища

$$
\mathfrak{M}=\left(\begin{array}{lll}
m_{1}^{-1} & m_{1}^{0} & m_{1}^{1} \\
m_{2}^{-1} & m_{2}^{0} & m_{2}^{1} \\
m_{3}^{-1} & m_{3}^{0} & m_{3}^{1}
\end{array}\right)
$$


таковы, что их элементы обладают следующими свойствами:

1) $m_{i}$ и $m_{i}^{j}$ - неотрицательные целые числа при всех значениях индексов $i \in$ $\{1,2,3\}, j \in\{-1,0,1\}$

2) для каждого $i \in\{1,2,3\}$ выполнено равенство $\sum_{j \in\{-1,0,1\}} m_{i}^{j}=m_{i}$;

3) для каждого $j \in\{-1,0,1\}$ вьполнено равенство $\sum_{i=1}^{3} m_{i}^{j}=l_{j}$;

4) $m_{1}+m_{2}+m_{3}=\sum_{i=1}^{3} \sum_{j \in\{-1,0,1\}} m_{i}^{j}=d$;

5) положим

$$
\begin{aligned}
k_{i}= & \operatorname{diam}^{2}\left\{\mathbf{v}=\left(v_{1}, \ldots, v_{m_{i}}\right):\right. \\
\quad & \left.\quad \operatorname{card}\left\{\nu=1, \ldots, m_{i}: v_{\nu}=j\right\}=m_{i}^{j} \quad \forall j \in\{-1,0,1\}\right\}, \quad i=1,2,3
\end{aligned}
$$

(корректность определения величины $k_{i}$ вытекает из уже постулированных свойств 1)-4)); тогда $k_{1}+k_{2}+k_{3}<k$.

Пусть, далее, $D_{3}=P\left(m_{1}, m_{2}, m_{3}\right), L_{3}=P\left(m_{1}^{-1}, m_{2}^{-1}, m_{3}^{-1}\right) \times P\left(m_{1}^{0}, m_{2}^{0}, m_{3}^{0}\right) \times$ $P\left(m_{1}^{1}, m_{2}^{1}, m_{3}^{1}\right)$ и $S_{3}=P\left(l_{-1}, l_{0}, l_{1}\right)$. Заметим, что определение параметра $S_{3}$ в данном частном случае полностью совпадает с определением величины $S$ в выражении (1).

Пусть теперь $\mathcal{X}=\left\{\left(x_{1}, x_{2}\right)\right\}$ - множество всех (упорядоченных) пар неотрицательных целых чисел $x_{1} \leqslant l_{1}$ и $x_{2} \leqslant l_{-1}$, для которых, в свою очередь, найдется пара $(-1,0,1)$-векторов $\mathbf{v}_{1}=\left(v_{1}^{1}, \ldots, v_{1}^{d-x_{1}-x_{2}}\right), \mathbf{v}_{2}=\left(v_{2}^{1}, \ldots, v_{2}^{d-x_{1}-x_{2}}\right) \in$ $\mathbb{R}^{d-x_{1}-x_{2}}$, удовлетворяюшая условиям:

(i) $\left(v_{i}^{1}\right)^{2}+\cdots+\left(v_{i}^{d-x_{1}-x_{2}}\right)^{2}=\left(l_{1}-x_{1}\right)+\left(l_{-1}-x_{2}\right), v_{i}^{1}+\cdots+v_{i}^{d-x_{1}-x_{2}}=$ $\left(l_{1}-x_{1}\right)-\left(l_{-1}-x_{2}\right), i=1,2$, т. е. каждый вектор $\mathbf{v}_{i}$ имеет $l_{1}-x_{1}$ положительных и $l_{-1}-x_{2}$ отрицательных компонент;

(ii) выполнены соотношения

$$
\left\{\nu: v_{1}^{\nu}=1\right\} \cap\left\{\nu: v_{2}^{\nu}=1\right\}=\varnothing, \quad\left\{\nu: v_{1}^{\nu}=-1\right\} \cap\left\{\nu: v_{2}^{\nu}=-1\right\}=\varnothing
$$

(iii) имеет место неравенство $\left|\mathbf{v}_{1}-\mathbf{v}_{2}\right|^{2} \leqslant k$.

Аналогично той части предыдущего пункта, в которой мы рассматривали проблему Грюнбаума, введем две группы параметров со штрихами. Элементы первой групшы будут зависеть от того, какую пару $\left(x_{1}, x_{2}\right) \in \mathcal{X}$ мы выберем и зафиксируем. В самом деле, положим $d^{\prime}=d-x_{1}-x_{2}, l_{1}^{\prime}=l_{1}-x_{1}, l_{-1}^{\prime}=l_{-1}-x_{2}, l_{0}^{\prime}=l_{0}$ и будем считать, что $\mathfrak{m}^{\prime}$ и $\mathfrak{M}^{\prime}$ суть (произвольные) вектор и матрища, обладаюшие всеми свойствами 1)-5) при формальной замене в них параметров без штрихов одноименными параметрами со штрихами. Аналогично (проведя формальную замену параметров) зададим величины $D_{3}^{\prime}, L_{3}^{\prime}, S_{3}^{\prime}$. В то же время положим $q_{i}=\min _{\left(x_{1}, x_{2}\right) \in \mathcal{X}} x_{i}, i=1,2$, и рассмотрим произвольные $p_{i} \in \mathbb{Z}: 0 \leqslant p_{i} \leqslant q_{i}$. От фиксации чисел $p_{i}$ будут, в свою очередь, зависеть элементы второй групшы параметров. Действительно, пусть $d^{\prime \prime}=d-p_{1}-p_{2}, l_{1}^{\prime \prime}=l_{1}-p_{1}, l_{-1}^{\prime \prime}=l_{-1}-p_{2}$, $l_{0}^{\prime \prime}=l_{0}$, a $\mathfrak{m}^{\prime \prime}$ и $\mathfrak{M}^{\prime \prime}$ отвечают этим параметрам в смысле выполнения свойств 1)-5). Стандартным образом зададим величины $D_{3}^{\prime \prime}, L_{3}^{\prime \prime}, S_{3}^{\prime \prime}$. Имеют место следующие теоремы. 
Теорема 6. Выполнена оценка

$$
f\left(l_{-1}, l_{0}, l_{1} ; k ; d\right) \leqslant \min _{\mathfrak{m}, \mathfrak{M}} G\left(D_{3}, L_{3}, S_{3}\right)
$$

Здесь минимум берется по всем возможнимм наборам параметров $\mathfrak{m}$ и $\mathfrak{M}$, удовлетворяющих свойствам 1)-5).

Tеорема 7. Выполнена оценка

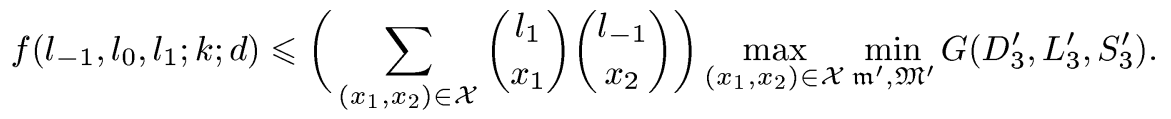

Здесь минимум берется по всем возможнним наборам параметров $\mathfrak{m}^{\prime}=$ $\mathfrak{m}^{\prime}\left(x_{1}, x_{2}\right)$ и $\mathfrak{M}^{\prime}=\mathfrak{M}^{\prime}\left(x_{1}, x_{2}\right)$, удовлетворяющих свойствам 1)-5).

ТЕОрема 8. Выполнена оценка

$$
f\left(l_{-1}, l_{0}, l_{1} ; k ; d\right) \leqslant \min _{p_{1}, p_{2}}\left(\begin{array}{c}
l_{1} \\
p_{1}
\end{array}\right)\left(\begin{array}{c}
l_{-1} \\
p_{2}
\end{array}\right) \min _{\mathfrak{m}^{\prime \prime}, \mathfrak{M}^{\prime \prime}} G\left(D_{3}^{\prime \prime}, L_{3}^{\prime \prime}, S_{3}^{\prime \prime}\right)
$$

Здесь минимум берется сначала по всем допустимым значениям величин $p_{1}, p_{2}$, а затем по всем возможнымм отвечающим им наборам параметров $\mathfrak{m}^{\prime \prime}$ и $\mathfrak{M}^{\prime \prime}$, удовлетворяющих свойствам 1)-5).

ЗАмЕчАниЕ 2. В специальных случаях удается уточнить выражения, стоящие в правых частях неравенств (9) и (10). Может так получиться, что какие-то из величин $p_{1}, p_{2}, x_{1}$ и $x_{2}$ окажутся равными своим максимально допустимым значениям: например, $x_{1}=l_{1}$ или $p_{2}=l_{-1}$. Тогда параметры типа $l_{1}^{\prime}$ обратятся в нуль. Пусть $x_{1}=l_{1}, x_{2}=l_{-1}$. В такой ситуации в правой части неравенства $(9)$ вместо $G\left(D_{3}^{\prime}, L_{3}^{\prime}, S_{3}^{\prime}\right)$ можно просто поставить единицу. Если $x_{2}=l_{-1}$, а $x_{1}<l_{1}$, то $D_{3}^{\prime}$, $L_{3}^{\prime}, S_{3}^{\prime}$ разумно заменить величинами $D, L, S$, которые получаются таким же образом, что и величины $D_{1}, L_{1}, S_{1}$ в теоремах 2 и 3 ; конечно, здесь уже следует апеллировать к нынешним параметрам $d^{\prime}, l_{1}^{\prime}$ и $l_{0}^{\prime}$. Аналогично необходимо поступить при $x_{1}=l_{1}, p_{1}=l_{1}$ и т. д.

ЗАмЕчАнИЕ 3 . Ясно, что теорема 6 является частным случаем теоремы 8. Мы сформулировали ее, поскольку во многих ситуациях она оказывается сильнее своего уточнения при $p_{1}+p_{2}>0$. Однако само уточнение весьма эффективно, и именно в нем отражается во всей полноте специфика проблемы Борсука, в то время как результат теоремы 6 имеет несколько более общий характер (например, он применим для нахождения оценок так называемых хроматических чисел хадвигеровых графов; см. [21]- [25]). Теорема 7, в свою очередь, в значительной мере усиливает как теорему 6 , так и теорему 8 . В $\S 6$ мы приведем различные примеры, подтверждающие сказанное.

Проблема Грюнбаума. Формулировки трех следуюших теорем аналогичны формулировкам теорем 6-8. Параметры $\mathcal{X}, q_{1}, q_{2}, p_{1}, p_{2}, d^{\prime}, d^{\prime \prime}, l_{-1}^{\prime}, l_{-1}^{\prime \prime}, l_{0}^{\prime}$, $l_{0}^{\prime \prime}, l_{1}^{\prime}, l_{1}^{\prime \prime}$ остаются неизменными, однако векторы $\mathfrak{g}, \mathfrak{g}^{\prime}, \mathfrak{g}^{\prime \prime}$ и матрищы $\mathfrak{G}, \mathfrak{G}^{\prime}, \mathfrak{G}^{\prime \prime}$, 
также возникающие в этой задаче, имеют несколько иной вид и удовлетворяют несколько иным условиям. Разумеется, мы выпишем эти условия лишь для $\mathfrak{g}$ и $\mathfrak{G}$, предполагая, как обычно, что остальные вариации получаются из указанных формальной заменой параметров со штрихами на параметры без штрихов.

Итак, вектор $\mathfrak{g}=\left(g_{-1}, g_{-1 / 2}, g_{0}, g_{1 / 2}, g_{1}\right)$ и матрица

$$
\mathfrak{G}=\left(\begin{array}{ccc}
g_{-1}^{-1} & g_{-1}^{0} & g_{-1}^{1} \\
g_{-1 / 2}^{-1} & g_{-1 / 2}^{0} & g_{-1 / 2}^{1} \\
g_{0}^{-1} & g_{0}^{0} & g_{0}^{1} \\
g_{1 / 2}^{-1} & g_{1 / 2}^{0} & g_{1 / 2}^{1} \\
g_{1}^{-1} & g_{1}^{0} & g_{1}^{1}
\end{array}\right)
$$

таковы, что:

1) $g_{i}$ и $g_{i}^{j}$ - неотрицательные целые числа при всех значениях индексов $i \in$ $\{-1,-1 / 2,0,1 / 2,1\}, j \in\{-1,0,1\}$;

2) для каждого $i \in\{-1,-1 / 2,0,1 / 2,1\}$ выполнено равенство $g_{i}^{-1}+g_{i}^{0}+g_{i}^{1}=g_{i}$;

3) для каждого $j \in\{-1,0,1\}$ вьполнено равенство $g_{-1}^{j}+g_{-1 / 2}^{j}+g_{0}^{j}+g_{1 / 2}^{j}+$ $g_{1}^{j}=l_{j}$;

4) $g_{-1}+g_{-1 / 2}+g_{0}+g_{1 / 2}+g_{1}=\sum_{i \in\{-1, \ldots, 1\}} \sum_{j \in\{-1,0,1\}} g_{i}^{j}=d$;

5) $\sum_{i \in\{-1, \ldots, 1\}} \sum_{j \in\{-1,0,1\}}(i-j)^{2} g_{i}^{j} \leqslant \frac{k}{4}$.

Заметим, что, как и в случае с $(0,1)$-многогранниками, верхние индексы элементов матрицы отвечают допустимым значениям координат векторов в любой из совокупностей вершин, подлежаших покрытию шарами; аналогично, нижние индексы можно интерпретировать как полусуммы таких значений. Положим

$$
\begin{aligned}
D_{4}= & P\left(g_{-1}, g_{-1 / 2}, g_{0}, g_{1 / 2}, g_{1}\right), \\
L_{4}= & P\left(g_{-1}^{-1}, g_{-1 / 2}^{-1}, g_{0}^{-1}, g_{1 / 2}^{-1}, g_{1}^{-1}\right) \times P\left(g_{-1}^{0}, g_{-1 / 2}^{0}, g_{0}^{0}, g_{1 / 2}^{0}, g_{1}^{0}\right) \\
& \times P\left(g_{-1}^{1}, g_{-1 / 2}^{1}, g_{0}^{1}, g_{1 / 2}^{1}, g_{1}^{1}\right), \\
S_{4}= & S_{3}=P\left(l_{-1}, l_{0}, l_{1}\right) .
\end{aligned}
$$

Соответственно определим параметры с одним и двумя штрихами. Имеют место следующие теоремы.

ТЕОрема 9. Выполнена оценка

$$
g\left(l_{-1}, l_{0}, l_{1} ; k ; d\right) \leqslant \min _{\mathfrak{g}, \mathfrak{G}} G\left(D_{4}, L_{4}, S_{4}\right) .
$$

Здесь минимум берется по всем возможнимм наборам параметров $\mathfrak{g} u \mathfrak{G}$, удовлетворяющих свойствам 1)-5).

TЕорема 10. Выполнена оценка

$$
g\left(l_{-1}, l_{0}, l_{1} ; k ; d\right) \leqslant\left(\sum_{\left(x_{1}, x_{2}\right) \in \mathcal{X}}\left(\begin{array}{c}
l_{1} \\
x_{1}
\end{array}\right)\left(\begin{array}{c}
l_{-1} \\
x_{2}
\end{array}\right)\right) \max _{\left(x_{1}, x_{2}\right) \in \mathcal{X}} \min _{\mathfrak{g}^{\prime}, \mathfrak{G}^{\prime}} G\left(D_{4}^{\prime}, L_{4}^{\prime}, S_{4}^{\prime}\right) .
$$

Здесь минимум берется по всем возможним наборам параметров $\mathfrak{g}^{\prime}=$ $\mathfrak{g}^{\prime}\left(x_{1}, x_{2}\right)$ и $\mathfrak{G}^{\prime}=\mathfrak{G}^{\prime}\left(x_{1}, x_{2}\right)$, удовлетворяющих свойствам 1)-5). 
ТЕОРема 11. Выполнена оценка

$$
g\left(l_{-1}, l_{0}, l_{1} ; k ; d\right) \leqslant \min _{p_{1}, p_{2}}\left(\begin{array}{c}
l_{1} \\
p_{1}
\end{array}\right)\left(\begin{array}{c}
l_{-1} \\
p_{2}
\end{array}\right) \min _{\mathfrak{g}^{\prime \prime}, \mathfrak{G}^{\prime \prime}} G\left(D_{4}^{\prime \prime}, L_{4}^{\prime \prime}, S_{4}^{\prime \prime}\right)
$$

Здесь минимум берется сначала по всем допустимым значениям величин $p_{1}, p_{2}$, а затем по всем возможнным отвечающим им наборам параметров $\mathfrak{g}^{\prime \prime}$ $u \mathfrak{G}^{\prime \prime}$, удовлетворяющ, их свойствам 1)-5).

Для теорем 9-11 можно сформулировать полные аналоги замечаний 2 и 3 . Как и в случае с задачей Борсука, мы обсудим второй из этих аналогов в 6 .

3.3. Общий случай. Рассмотрим отдельно проблему Борсука и отдельно проблему Грюнбаума.

Проблема Борсука. Наконец, мы готовы дать общую формулировку результата, применимую не только для $(0,1)$-многогранников и кросс-политопов, но и для многогранников общего вида. В действительности, такая формулировка будет одна, но она фактически будет сочетать в себе все элементы прежних теорем: с одной стороны, ее, несмотря на громоздкость, будет значительно легче понять, а с другой стороны, с ее помощью можно будет получить максимально эффективные оценки. Итак, необходимо последовательно ввести достаточно много параметров, по которым будет производиться оптимизация.

Рассмотрим множество $\mathcal{X}$ наборов неотрицательных целых чисел $\left(x_{1}, \ldots, x_{t}\right)$, считая, что набор $\left(x_{1}, \ldots, x_{t}\right)$ принадлежит $\mathcal{X}$ в том и только в том случае, когда выполнены следуюшие условия:

а) $0 \leqslant x_{1} \leqslant l_{a_{1}}, \ldots, 0 \leqslant x_{t} \leqslant l_{a_{t}}$ и, значит, $x_{1}+\cdots+x_{t} \leqslant d$;

b) исключая из множества $\left\{a_{1}, \ldots, a_{t}\right\}$ те его элементы $a_{j}$, которые удовлетворяют равенству $l_{a_{j}}=x_{j}$, получая, тем самым, новое множество $\left\{\alpha_{1}, \ldots, \alpha_{\tau}\right\} \subseteq$ $\left\{a_{1}, \ldots, a_{t}\right\}, \tau \leqslant t$, и полагая, кроме того, $d^{\prime}=d-x_{1}-\cdots-x_{t}$ и $l_{a_{j}}^{\prime}=l_{a_{j}}-x_{j}$, $j=1, \ldots, t$, имеем: найдется пара векторов $\mathbf{v}_{1}=\left(v_{1}^{1}, \ldots, v_{1}^{d^{\prime}}\right), \mathbf{v}_{2}=\left(v_{2}^{1}, \ldots, v_{2}^{d^{\prime}}\right) \in$ $\mathbb{R}^{d^{\prime}}$ таких, что card $\left\{\nu=1, \ldots, d^{\prime}: v_{i}^{\nu}=\alpha_{j}\right\}=l_{\alpha_{j}}^{\prime}, i=1,2, j=1, \ldots, \tau$, при этом одновременно $v_{1}^{\nu} \neq v_{2}^{\nu}, \nu=1, \ldots, d^{\prime}$, и $\left|\mathbf{v}_{1}-\mathbf{v}_{2}\right|^{2} \leqslant k$.

Ясно, что данное определение корректно: $d^{\prime}=l_{\alpha_{1}}^{\prime}+\cdots+l_{\alpha_{\tau}}^{\prime}$ и $l_{\alpha_{j}}^{\prime}>0$ (последнее неравенство необходимо для того, чтобы векторы $\mathbf{v}_{1}, \mathbf{v}_{2}$ имели координаты, равные $\alpha_{j}$, т. е., иными словами, чтобы параметры $l_{\alpha_{1}}^{\prime}, \ldots, l_{\alpha_{\tau}}^{\prime}$ играли для векторов размерности $d^{\prime}$ в точности ту же роль, какую параметры $l_{a_{1}}>0, \ldots, l_{a_{t}}>0$ играют для векторов размерности $d$; ср. замечание 2 ).

Положим теперь $q_{i}=\min _{\left(x_{1}, \ldots, x_{t}\right) \in \mathcal{X}} x_{i}, i=1,2, \ldots, t$, и для произвольной последовательности натуральных чисел $1 \leqslant \nu_{1}<\cdots<\nu_{\theta} \leqslant t, 0 \leqslant \theta \leqslant t$ (при $\theta=0$ последовательность, естественно, пуста), определим множество $\widetilde{\mathcal{X}}=\widetilde{\mathcal{X}}_{\nu_{1}, \ldots, \nu_{\theta}}$ таких наборов чисел $\left(x_{\nu_{1}}, \ldots, x_{\nu_{\theta}}\right)$, что существует набор $\left(x_{1}, \ldots, x_{t}\right) \in \mathcal{X}$, в котором на позищиях с номерами $\nu_{1}, \ldots, \nu_{\theta}$ стоят числа $x_{\nu_{1}}, \ldots, x_{\nu_{\theta}}$. Пусть множество $\left\{\mu_{1}, \ldots, \mu_{t-\theta}\right\}$ (возможно, пустое) задается равенством $\left\{\mu_{1}, \ldots, \mu_{t-\theta}\right\}=$ $\{1, \ldots, t\} \backslash\left\{\nu_{1}, \ldots, \nu_{\theta}\right\}$. Рассмотрим каждый набор чисел $x_{\nu_{1}}, \ldots, x_{\nu_{\theta}}$ и дополним его каждым (произвольным) набором таких целых чисел $p_{\mu_{1}}, \ldots, p_{\mu_{t-\theta}}$, что $0 \leqslant$ $p_{\mu_{j}} \leqslant q_{\mu_{j}}$. Обозначим через $\left(\eta_{1}, \ldots, \eta_{t}\right)$ упорядоченный набор чисел $x_{\nu_{1}}, \ldots, x_{\nu_{\theta}}$, 
$p_{\mu_{1}}, \ldots, p_{\mu_{t-\theta}}$. Аналогично тому, как это было сделано при формулировке условия b), перейдем от множества $\left\{a_{1}, \ldots, a_{t}\right\}$ к множеству $\left\{\alpha_{1}, \ldots, \alpha_{\tau}\right\} \subseteq\left\{a_{1}, \ldots\right.$ $\left.\ldots, a_{t}\right\}, \tau \leqslant t$, последовательно удаляя те $a_{j}$, для которых $l_{a_{j}}=\eta_{j}$. Положим также $d^{\prime}=d-\eta_{1}-\cdots-\eta_{t}$ и $l_{a_{j}}^{\prime}=l_{a_{j}}-\eta_{j}, j=1, \ldots, t$. Понятно, что $d^{\prime}=l_{\alpha_{1}}^{\prime}+\cdots$ $\cdots+l_{\alpha_{\tau}}^{\prime}$, a $l_{\alpha_{j}}^{\prime}>0, j=1, \ldots, \tau$.

Все вводимые далее параметры будут определяться сообразно сделанному выбору величин $\alpha_{1}, \ldots, \alpha_{\tau}, l_{\alpha_{1}}^{\prime}, \ldots, l_{\alpha_{\tau}}^{\prime}$ и $d^{\prime}$, т.е., в конечном счете, они будут находиться в некоторой зависимости от того, как мы фиксировали числа $\eta_{1}, \ldots, \eta_{t}$. С целью уменьшения громоздкости обозначений мы эту зависимость явно указывать не будем.

Итак, введем еще одну группу параметров: вектор $\mathfrak{m}=\left(m_{1}, \ldots, m_{\tau}\right)$ и матрищу

$$
\mathfrak{M}=\left(\begin{array}{cccc}
m_{1}^{\alpha_{1}} & m_{1}^{\alpha_{2}} & \ldots & m_{1}^{\alpha_{\tau}} \\
m_{2}^{\alpha_{1}} & m_{2}^{\alpha_{2}} & \ldots & m_{2}^{\alpha_{\tau}} \\
\ldots & \ldots & \ldots & \ldots \\
m_{\tau}^{\alpha_{1}} & m_{\tau}^{\alpha_{2}} & \ldots & m_{\tau}^{\alpha_{\tau}}
\end{array}\right)
$$

элементы которых обладают следующими свойствами:

1) $m_{i}$ и $m_{i}^{\alpha_{j}}$ - неотрицательные целые числа при всех значениях индексов $i, j=$ $1, \ldots, \tau$

2) для каждого $i \in\{1, \ldots, \tau\}$ выполнено равенство $\sum_{j=1}^{\tau} m_{i}^{\alpha_{j}}=m_{i}$;

3) для каждого $j \in\{1, \ldots, \tau\}$ выполнено равенство $\sum_{i=1}^{\tau} m_{i}^{\alpha_{j}}=l_{\alpha_{j}}^{\prime}$;

4) $m_{1}+\cdots+m_{\tau}=\sum_{i, j=1}^{\tau} m_{i}^{\alpha_{j}}=d^{\prime}$;

5) положим

$$
\begin{aligned}
k_{i}= & \operatorname{diam}^{2}\left\{\mathbf{v}=\left(v_{1}, \ldots, v_{m_{i}}\right):\right. \\
& \left.\operatorname{card}\left\{\nu=1, \ldots, m_{i}: v_{\nu}=\alpha_{j}\right\}=m_{i}^{\alpha_{j}} \quad \forall j \in\{1, \ldots, \tau\}\right\}, \quad i=1, \ldots, \tau
\end{aligned}
$$

(корректность определения величины $k_{i}$ вытекает из уже постулированных свойств 1)-4)); тогда $k_{1}+\cdots+k_{\tau}<k$.

Пусть, наконец, $D_{5}=P\left(m_{1}, \ldots, m_{\tau}\right), L_{5}=\prod_{j=1}^{\tau} P\left(m_{1}^{\alpha_{j}}, \ldots, m_{\tau}^{\alpha_{j}}\right)$, а $S_{5}$ задается, по сушеству, формулой $(1)$, т. е. $S_{5}=P\left(l_{\alpha_{1}}^{\prime}, \ldots, l_{\alpha_{\tau}}^{\prime}\right)$. Имеет место

Tеорема 12. Пусть $\varphi_{j}=a_{\nu_{j}}, j=1, \ldots, \theta, \quad \psi_{j}=a_{\mu_{j}}, j=1, \ldots, t-\theta$. Виполнена оченка

$$
\begin{aligned}
& f\left(l_{a_{1}}, \ldots, l_{a_{t}} ; k ; d\right) \leqslant \min _{\theta} \min _{\nu_{1}, \ldots, \nu_{\theta}}\left(\sum_{\left(x_{\nu_{1}}, \ldots, x_{\nu_{\theta}}\right) \in \tilde{\mathcal{X}}}\left(\begin{array}{c}
l_{\varphi_{1}} \\
x_{\nu_{1}}
\end{array}\right) \times \cdots \times\left(\begin{array}{c}
l_{\varphi_{\theta}} \\
x_{\nu_{\theta}}
\end{array}\right)\right) \\
& \times \max _{\left(x_{\nu_{1}}, \ldots, x_{\nu_{\theta}}\right) \in \widetilde{\mathcal{X}} p_{\mu_{1}}, \ldots, p_{\mu_{t-\theta}}} \min _{\mathfrak{m}, \mathfrak{M}}\left(\begin{array}{c}
l_{\psi_{1}} \\
p_{\mu_{1}}
\end{array}\right) \times \cdots \times\left(\begin{array}{c}
l_{\psi_{t-\theta}} \\
p_{\mu_{t-\theta}}
\end{array}\right) \\
& \times G\left(D_{5}, L_{5}, S_{5}\right) \text {. }
\end{aligned}
$$

Здесь в первой части произведения минимум берется сначала по всем $\theta \in$ $\{0, \ldots, t\}$, а затем по всем последовательностям $\nu_{1}, \ldots, \nu_{\theta}$, если таковые 
существуют. При $\theta=0$ остаются лишь величины типа р и все обгекты, которые от них зависят. Во второй части произведения сначала независимо берутся максимум и минимум по параметрам $x_{\nu_{1}}, \ldots, x_{\nu_{\theta}} u p_{\mu_{1}}, \ldots, p_{\mu_{t-\theta}}$ соответственно (при $\theta=0 u \theta=t$ один из этих әкстремумов пропадает, и в дальнейшем остается зависимость только от параметров одного типа); затем производится минимизация по всем возможным наборам параметров $\mathfrak{m}$ и М̆, обладающих свойствами 1)-5) и отвечающих, в свою очередь, параметрам $\eta_{1}, \ldots, \eta_{t}$.

ЗАмЕчАнИЕ 4. Установить связь между теоремой 12 и, скажем, теоремами 6-8 нетрудно. Например, теорема 7 справедлива при $\theta=2$ и $p_{\mu_{1}}=0(t=3)$. Теорема 8 имеет место, коль скоро $\theta=0, p_{\mu_{1}}=p_{1}, p_{\mu_{2}}=p_{2}$ и $p_{\mu_{3}}=0$. Верхняя и нижняя индексации параметров в матрице $\mathfrak{M}$ вполне коррелируют с аналогичной индексацией параметров в одноименных матрицах из предшествующих пунктов.

Проблема Грюнбаума. Как и в предыдущей части пункта, введем некоторые

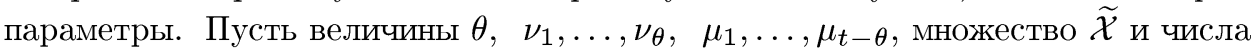
$\eta_{1}, \ldots, \eta_{t}, \alpha_{1}, \ldots, \alpha_{\tau}, l_{\alpha_{1}}^{\prime}, \ldots, l_{\alpha_{\tau}}^{\prime}, d^{\prime}$ построены точно так же, как это было сделано для проблемы Борсука. Рассмотрим дополнительно множество

$$
\left\{\beta_{1}, \ldots, \beta_{\rho}\right\}=\left\{\frac{\alpha_{i}+\alpha_{j}}{2}, i, j=1, \ldots, \tau\right\}
$$

здесь $\beta_{\nu} \neq \beta_{\mu}, \quad \nu \neq \mu, \nu, \mu \in\{1, \ldots, \rho\}$, так что заведомо $\tau \leqslant \rho \leqslant \frac{\tau(\tau+1)}{2}$ (ясно, что $\frac{\alpha_{i}+\alpha_{j}}{2}=\frac{\alpha_{j}+\alpha_{i}}{2}$ и $\alpha_{i} \in\left\{\beta_{1}, \ldots, \beta_{\rho}\right\}$ для любого $\left.i\right)$. Как и в случае теоремы 12 , зададим теперь параметры $\mathfrak{g}=\left\{g_{\beta_{1}}, \ldots, g_{\beta_{\rho}}\right\}$ и

$$
\mathfrak{G}=\left(\begin{array}{cccc}
g_{\beta_{1}}^{\alpha_{1}} & g_{\beta_{1}}^{\alpha_{2}} & \ldots & g_{\beta_{1}}^{\alpha_{\tau}} \\
g_{\beta_{2}}^{\alpha_{1}} & g_{\beta_{2}}^{\alpha_{2}} & \ldots & g_{\beta_{2}}^{\alpha_{\tau}} \\
\ldots & \ldots & \ldots & \ldots \\
g_{\beta_{\rho}}^{\alpha_{1}} & g_{\beta_{\rho}}^{\alpha_{2}} & \ldots & g_{\beta_{\rho}}^{\alpha_{\tau}}
\end{array}\right)
$$

предполагая, что для них формально выполнены свойства 1)-4) параметров $\mathfrak{m}$ и $\mathfrak{M}$ первой части настоящего пункта; при этом, естественно, величины $m_{i}$ и $m_{i}^{\alpha_{j}}$, $i, j=1, \ldots, \tau$, фигурирующие в формулировках свойств, нужно заменить величинами $g_{\beta_{i}}$ и $g_{\beta_{i}}^{\alpha_{j}}, i=1, \ldots, \rho, j=1, \ldots, \tau$. Кроме того, должно иметь место новое свойство

5) $\sum_{i=1}^{\rho} \sum_{j=1}^{\tau}\left(\beta_{i}-\alpha_{j}\right)^{2} g_{\beta_{i}}^{\alpha_{j}} \leqslant \frac{k}{4}$.

Легко видеть, что новые параметры $\mathfrak{g}$ и $\mathfrak{G}$ полностью коррелируют с одноименными параметрами из предыдущих пунктов. Понятно, что в следующей теореме, подобно теореме 12 , будут использованы все результаты, полученные нами в частных случаях. Пусть, наконец, $D_{6}=P\left(g_{\beta_{1}}, \ldots, g_{\beta_{\rho}}\right), L_{6}=\prod_{j=1}^{\tau} P\left(g_{\beta_{1}}^{\alpha_{j}}, \ldots, g_{\beta_{\rho}}^{\alpha_{j}}\right)$, a $S_{6}=S_{5}=P\left(l_{\alpha_{1}}^{\prime}, \ldots, l_{\alpha_{\tau}}^{\prime}\right)$. Имеет место 
Teopema 13. Пусть $\varphi_{j}=a_{\nu_{j}}, \quad j=1, \ldots, \theta, \quad \psi_{j}=a_{\mu_{j}}, \quad j=1, \ldots, t-\theta$. Выполнена оченка

$$
\begin{aligned}
g\left(l_{a_{1}}, \ldots,\right. & \left.l_{a_{t}} ; k ; d\right) \leqslant \min _{\theta} \min _{\nu_{1}, \ldots, \nu_{\theta}}\left(\sum_{\left(x_{\nu_{1}}, \ldots, x_{\nu_{\theta}}\right) \in \tilde{\mathcal{X}}}\left(\begin{array}{c}
l_{\varphi_{1}} \\
x_{\nu_{1}}
\end{array}\right) \times \cdots \times\left(\begin{array}{c}
l_{\varphi_{\theta}} \\
x_{\nu_{\theta}}
\end{array}\right)\right) \\
& \times \max _{\left(x_{\nu_{1}}, \ldots, x_{\nu_{\theta}}\right) \in \tilde{\mathcal{X}}} \min _{p_{\mu_{1}}, \ldots, p_{\mu_{t-\theta}}} \min _{\mathfrak{g}, \mathfrak{G}}\left(\begin{array}{c}
l_{\psi_{1}} \\
p_{\mu_{1}}
\end{array}\right) \times \cdots \times\left(\begin{array}{c}
l_{\psi_{t-\theta}} \\
p_{\mu_{t-\theta}}
\end{array}\right) \\
& \times G\left(D_{6}, L_{6}, S_{6}\right) .
\end{aligned}
$$

Здесь оптимизация проводится точно так же, как и в теореме 12.

Теоремы 4, 5 и 9-11 несложно выводятся как частные случаи из теоремы 13. Естественно, делается это точно так же, как и для проблемы Борсука (см. замечание 4).

\section{§4. Вспомогательные определения: задача о покрытии}

Предваряя доказательства новых теорем, мы дадим в настоящем параграфе некоторые определения и сформулируем некоторые вспомогательные результаты, которые в этих доказательствах будут использованы. Все эти определения и результаты связаны с так называемой задачей о покрытии, или, точнее, с задачей о нахождении минимальной системы общих представителей для совокупности конечных множеств.

Итак, мы рассматриваем произвольное конечное множество $\Re_{D}=\{1, \ldots, D\}$, состояшее из $D$ элементов. В этом множестве мы выбираем произвольную совокупность подмножеств $\mathcal{M}=\left\{M_{1}, \ldots, M_{S}\right\} \subset 2^{\Re_{D}}$, предполагая в то же время, что нам известна (универсальная) нижняя оценка мощности каждого из подмножеств $M_{i} \in \mathcal{M}: \operatorname{card} M_{i} \geqslant L$. Для такой совокупности множеств мы определяем систему общих представителей (с. о.п.) как еще одно подмножество $R \subset \Re_{D}$, которое содержит хотя бы по одному элементу ("представителю") из каждого $M_{i} \in \mathcal{M}$, т. е. $R \cap M_{i} \neq \varnothing$ для любого $i$. Ясно, что сard $R \leqslant \min \{D, S\}$, поскольку, во-первых, множество $\Re_{D}$ заведомо является с. о.п. для любой совокупности $\mathcal{M} \subset 2^{\Re_{D}}$, а во-вторых $\operatorname{card} \mathcal{M}=S$. Понятно также, что с. о.п. $R$ определяется по $\mathcal{M}$, вообще говоря, неоднозначно. В простейшей формулировке задача о покрытии состоит в том, чтобы отыскать минимальную с. о.п. для $\mathcal{M}$. Мошность этой с. о. п. (тоже, конечно, не обязательно единственной) мы будем обозначать через $\zeta(\mathcal{M})$. Наконец, через $Z(D, L, S)$ мы обозначим величину $\max _{\mathcal{M}} \zeta(\mathcal{M})$, где максимум берется по всем совокупностям множеств $\mathcal{M}$ с параметрами $D, L, S$.

Основной результат о системах общих представителей состоит в том, что асимптотически величина $Z(D, L, S)$ ведет себя так же, как и определенная в предыдущем параграфе функция $G(D, L, S)$. Таким образом, может быть сформулирована следуюшая теорема.

TеОРема 14. При любых значениях параметров $D, L, S$ выполнена оченка $Z(D, L, S) \leqslant G(D, L, S)$. Если параметры удовлетворяют некоторым доста- 
точно общим соотношениям, то

$$
Z(D, L, S)=\frac{D}{L} \log \frac{S L}{D}+O\left(\frac{D}{L}\left(\log \log \frac{S L}{D}+\log \log L\right)\right)
$$

Далее нам потребуется лишь неравенство $Z(D, L, S) \leqslant G(D, L, S)$, поэтому пояснять в настоящей работе выражение “достаточно общие соотношения" мы не будем (хотя вид остаточного члена в (16) подсказывает, что выполнено соотношение $\log L=o\left(\frac{S L}{D}\right)$, при котором первый член асимптотики остается доминируюшим). Сама теорема была доказана в разное время несколькими авторами (см., например, [14], [28]-[32]). Нетривиальные усиления и вариации этой теоремы были также предложены автором в [33].

\section{§5. Доказательства результатов}

Пусть фиксированы все параметры в каждой из сформулированных теорем. В частности, будем считать заранее заданной совокупность вершин потенциального решетчатого многогранника - совокупность векторов $\mathcal{F}\left(l_{a_{1}}, \ldots, l_{a_{t}} ; k ; d\right)$, которую в той или иной ситуации нам предстоит либо разбить на надлежащее число частей меньшего диаметра, либо покрыть “меньшими” шарами. В дальнейшем нам удобнее будет рассматривать не совокупность $\mathcal{F}\left(l_{a_{1}}, \ldots, l_{a_{t}} ; k ; d\right)=\left\{\mathbf{v}_{1}, \ldots, \mathbf{v}_{s}\right\}$, $\mathbf{v}_{i}=\left(v_{i}^{1}, \ldots, v_{i}^{d}\right)$, а однозначно отвечающую ей совокупность (упорядоченных) разбиений

$$
\mathcal{M}\left(l_{a_{1}}, \ldots, l_{a_{t}} ; k ; d\right)=\left\{\left(M_{1}^{1}, \ldots, M_{1}^{t}\right), \ldots,\left(M_{s}^{1}, \ldots, M_{s}^{t}\right)\right\} \subset 2^{2^{\Re} d}
$$

множества $\Re_{d}=\{1, \ldots, d\}$ на $t$ таких частей, что сard $M_{i}^{j}=l_{a_{j}}, i=1, \ldots, s$, $j=1, \ldots, t: M_{i}^{j}=\left\{\nu \in \Re_{d}: v_{i}^{\nu}=a_{j}\right\}$.

Имея такую совокупность разбиений, мы подробно докажем самую общую теорему, например теорему 12 , а затем на более частных случаях проиллюстрируем безусловно громоздкое доказательство. С одной стороны, нам не придется многократно повторять похожие рассуждения, а с другой стороны, мы в конечном итоге добьемся лучшего понимания основных пружин (по существу, комбинаторных) доказательств. Разумеется, теорему 13 мы также подробно прокомментируем.

5.1. Доказательство теоремы 12. Чтобы сделать наши рассуждения более прозрачными, структурируем их, расписав по шагам.

Шаг 1. Имеет место следуюшее

УТВЕРЖДЕНИЕ. Рассмотрим произвольную пару разбиений $\left(M_{i}^{1}, \ldots, M_{i}^{t}\right)$, $\left(M_{j}^{1}, \ldots, M_{j}^{t}\right), \quad i \neq j, \quad i, j \in\{1, \ldots, s\}$, әлементы которой принадлежат совокупности $\mathcal{M}\left(l_{a_{1}}, \ldots, l_{a_{t}} ; k ; d\right)$. Положим $x_{1}=M_{i}^{1} \cap M_{j}^{1}, \ldots, x_{t}=M_{i}^{t} \cap M_{j}^{t}$. Тогда последовательность $\left(x_{1}, \ldots, x_{t}\right)$ входит в $\mathcal{X}$. 
ДокАЗАТЕЛЬСТво. Зададим множество $\Re$ путем удаления из множества $\Re_{d}=$ $\{1, \ldots, d\}$ попарных пересечений тех элементов разбиений, верхние индексы которых совпадают:

$$
\Re=\Re_{d} \backslash\left(M_{i}^{1} \cap M_{j}^{1}\right) \backslash \cdots \backslash\left(M_{i}^{t} \cap M_{j}^{t}\right) .
$$

Понятно, что новообразованное множество $\Re$ может быть естественно отождествлено с множеством $\Re_{d^{\prime}}$, где, в силу введенных обозначений, $d^{\prime}=d-x_{1}-\cdots-x_{t}$, и это коррелирует с тем параметром $d^{\prime}$, которьй мы ввели при формулировке теоремы. Далее, разбиения $\left(M_{i}^{1}, \ldots, M_{i}^{t}\right),\left(M_{j}^{1}, \ldots, M_{j}^{t}\right)$ легко преобразуются в разбиения $\left(\widetilde{M}_{i}^{1}, \ldots, \widetilde{M}_{i}^{t}\right),\left(\widetilde{M}_{j}^{1}, \ldots, \widetilde{M}_{j}^{t}\right)$ множества $\Re_{d^{\prime}}$ на части, имеюшие мошности $l_{a_{\xi}}^{\prime}, \xi=1, \ldots, t$, соответственно:

$$
\widetilde{M_{\nu}^{\xi}}=M_{\nu}^{\xi} \backslash\left(M_{i}^{\xi} \cap M_{j}^{\xi}\right), \quad \nu \in\{i, j\}, \quad \xi \in\{1, \ldots, t\} .
$$

При этом в разбиениях "с волной" могут возникнуть элементы нулевой мощности, так как известно, что $l_{a_{\xi}}^{\prime}=l_{a_{\xi}}-x_{\xi}$, а величина $x_{\xi}$, вообще говоря, достигает свое максимально допустимое значение $l_{a_{\xi}}$. Чтобы упростить запись, прибегнем к помощи известных нам обозначений и заменим разбиения $\left(\widetilde{M}_{i}^{1}, \ldots, \widetilde{M}_{i}^{t}\right)$, $\left(\widetilde{M}_{j}^{1}, \ldots, \widetilde{M}_{j}^{t}\right)$ множества $\Re_{d^{\prime}}$ разбиениями $\left(\bar{M}_{i}^{1}, \ldots, \bar{M}_{i}^{\tau}\right),\left(\bar{M}_{j}^{1}, \ldots, \bar{M}_{j}^{\tau}\right)$ того же множества на части, имеющие положительные мощности $l_{\alpha_{\xi}}^{\prime}, \xi=1, \ldots, \tau$.

Вспоминая теперь, как осуществлялось взаимно однозначное соответствие между векторами и разбиениями, перейдем от разбиений $\left(\bar{M}_{i}^{1}, \ldots, \bar{M}_{i}^{\tau}\right)$, $\left(\bar{M}_{j}^{1}, \ldots, \bar{M}_{j}^{\tau}\right)$ к векторам $\mathbf{v}_{1}=\left(v_{1}^{1}, \ldots, v_{1}^{d^{\prime}}\right), \mathbf{v}_{2}=\left(v_{2}^{1}, \ldots, v_{2}^{d^{\prime}}\right) \in \mathbb{R}^{d^{\prime}}$. Ясно, что $\operatorname{card}\left\{\nu=1, \ldots, d^{\prime}: v_{i}^{\nu}=\alpha_{\xi}\right\}=l_{\alpha_{\xi}}^{\prime}, \quad i=1,2, \quad \xi=1, \ldots, \tau$, и что в то же время $v_{1}^{\nu} \neq v_{2}^{\nu}, \nu=1, \ldots, d^{\prime}$, поскольку общие части элементов первоначальных разбиений, от которых мы избавились, отвечали совпадающим координатам того или иного вида в первоначальных векторах (размерности $d$ ). Понятно, наконец, что $\left|\mathbf{v}_{1}-\mathbf{v}_{2}\right|^{2} \leqslant k$, так как сумма квадратов разностей одинаковых координат, стоявших на уничтоженных нами $x_{1}+\cdots+x_{t}$ позищиях, никакого вклада в расстояние давать не могла и, следовательно, расстояния между первоначальными $d$-мерными векторами (лежашими в совокупности $\mathcal{F}\left(l_{a_{1}}, \ldots, l_{a_{t}} ; k ; d\right)$ диаметра $\left.\sqrt{k}\right)$ и векторами $\mathbf{v}_{1}, \mathbf{v}_{2}$ друг от друга не отличаются. Значит, для чисел $x_{1}, \ldots, x_{t}$ выполнены все условия а), b) из определения множества $\mathcal{X}$, и $\left(x_{1}, \ldots, x_{t}\right) \in \mathcal{X}$. Утверждение доказано.

Подытоживая полученное на первом шаге, заметим, что мы фактически прояснили смысл целой группы параметров: действительно, $\mathcal{X}$ представляет собой совокупность допустимых наборов пересечений множеств координат одного вида в разных векторах из $\mathcal{F}\left(l_{a_{1}}, \ldots, l_{a_{t}} ; k ; d\right)$. Основным определяюшим фактором при построении $\mathcal{X}$ служит соотношение между числами $l_{a_{1}}, \ldots, l_{a_{t}}$ и $k$. Более детально мы обсудим это в $\S 6$.

Шаг 2. Зафиксируем произвольное $\theta$, произвольные $\nu_{1}, \ldots, \nu_{\theta}$, отвечаюшие им $\mu_{1}, \ldots, \mu_{t-\theta}$ и любые $p_{\mu_{1}}, \ldots, p_{\mu_{t-\theta}}$ (см. формулировку теоремы). Положим

$$
D^{*}=\sum_{\left(x_{\nu_{1}}, \ldots, x_{\nu_{\theta}}\right) \in \tilde{\mathcal{X}}}\left(\begin{array}{c}
d \\
\eta_{1}
\end{array}\right) \times\left(\begin{array}{c}
d-\eta_{1} \\
\eta_{2}
\end{array}\right) \times \cdots \times\left(\begin{array}{c}
d-\eta_{1}-\cdots-\eta_{t-1} \\
\eta_{t}
\end{array}\right),
$$


где числа $\eta_{1}, \ldots, \eta_{t}$ определяются по фиксированным выше $p_{\mu_{1}}, \ldots, p_{\mu_{t-\theta}}$ и по

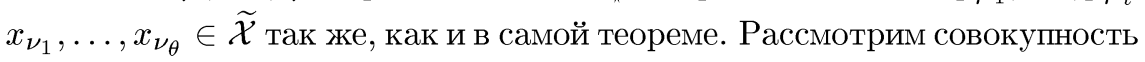

$$
\mathcal{K}=\left\{\left(K_{1}^{1}, \ldots, K_{1}^{t}\right), \ldots,\left(K_{D^{*}}^{1}, \ldots, K_{D^{*}}^{t}\right)\right\} \subset 2^{2^{\Re} d}
$$

состоящую из всевозможных (упорядоченных) наборов попарно непересекающихся множеств, имеющих мошности саrd $K_{i}^{j}=\eta_{j}, i=1, \ldots, D^{*}, j=1, \ldots, t$. Корректность такого определения очевидна благодаря свойствам параметров $\eta_{1}, \ldots$ $\ldots, \eta_{t}$, которые заведомо не превосходят $l_{a_{1}}, \ldots, l_{a_{t}}$ соответственно. Заметим, что, как правило, наборы из совокупности $\mathcal{K}$ разбиениями не являются, и в этом состоит их принципиальное отличие от наборов из основной совокупности $\mathcal{M}\left(l_{a_{1}}, \ldots\right.$ $\left.\ldots, l_{a_{t}} ; k ; d\right)$, которую мы рассматриваем.

Теперь совершим переход, который, как мы фактически увидим далее, можно считать двойственным. Сначала мы естественным способом сопоставим совокупности $\mathcal{K}$ множество $\Re_{D^{*}}$, т. е. каждый набор $\left(K_{i}^{1}, \ldots, K_{i}^{t}\right)$ будем кодировать своим порядковым номером $i$. Вместе с тем, всякому разбиению $\left(M_{j}^{1}, \ldots, M_{j}^{t}\right)$ из $\mathcal{M}\left(l_{a_{1}}, \ldots, l_{a_{t}} ; k ; d\right)$ (а значит, и всякому вектору из $\mathcal{F}\left(l_{a_{1}}, \ldots, l_{a_{t}} ; k ; d\right)$ ) поставим в соответствие подмножество $\Lambda_{j} \subset \Re_{D^{*}}$ :

$$
\Lambda_{j}=\left\{i \in \Re_{D^{*}}: K_{i}^{1} \subset M_{j}^{1}, \ldots, K_{i}^{t} \subset M_{j}^{t}\right\}
$$

Заметим, что мощность $L^{*}$ любого $\Lambda_{j}$ задается равенством

$$
L^{*}=\left(\sum_{\left(x_{\nu_{1}}, \ldots, x_{\nu_{\theta}}\right) \in \tilde{\mathcal{X}}}\left(\begin{array}{c}
l_{\varphi_{1}} \\
x_{\nu_{1}}
\end{array}\right) \times \cdots \times\left(\begin{array}{c}
l_{\varphi_{\theta}} \\
x_{\nu_{\theta}}
\end{array}\right)\right) \times\left(\begin{array}{c}
l_{\psi_{1}} \\
p_{\mu_{1}}
\end{array}\right) \times \cdots \times\left(\begin{array}{c}
l_{\psi_{t-\theta}} \\
p_{\mu_{t-\theta}}
\end{array}\right),
$$

где $\varphi_{1}, \ldots, \varphi_{\theta}$ и $\psi_{1}, \ldots, \psi_{t-\theta}$ - числа, являющиеся известными по формулировке теоремы сокращениями для чисел $a_{1}, \ldots, a_{t}$.

Итак, мы получили совокупность $\mathfrak{L}=\left\{\Lambda_{1}, \ldots, \Lambda_{s}\right\} \subset 2^{\Re} D^{*}$, каждый элемент которой имеет мощность $L^{*}$.

Выберем какую-нибудь минимальную с.о.п. $\left\{\lambda_{1}, \ldots, \lambda_{\zeta}\right\}, \zeta=\zeta(\mathfrak{L})$, для совокупности $\mathfrak{L}$. Чтобы оценить сверху величину $\zeta$ (а такая оценка понадобится), можно, в принципе, воспользоваться теоремой 14: в самом деле, параметры $D=D^{*}$ и $L=L^{*}$ уже известны, а в качестве $S=S^{*}$ можно взять универсальную оценку для $s=\operatorname{card} \mathcal{F}\left(l_{a_{1}}, \ldots, l_{a_{t}} ; k ; d\right)$, которую обеспечивает неравенство (1). Далее в аналогичных ситуациях мы именно так и поступим. Однако сейчас заметим, что верхняя граница мощности наименьшей с. о.п. для $\mathfrak{L}$ может быть найдена и другим путем. А именно, из доказанного на шаге 1 утверждения вытекает свойство: каковы бы ни были два множества $\Lambda_{i}, \Lambda_{j}$ из совокупности $\mathfrak{L}$, их пересечение обязательно непусто. Ясно тогда, что $\zeta \leqslant L^{*}$.

Основываясь на полученных результатах, образуем покрытие совокупности $\mathcal{M}\left(l_{a_{1}}, \ldots, l_{a_{t}} ; k ; d\right)$ меньшими, вообще говоря, совокупностями $\mathcal{M}_{1}, \ldots, \mathcal{M}_{\zeta}$ :

$$
\mathcal{M}_{i}=\left\{\left(M_{j}^{1}, \ldots, M_{j}^{t}\right) \in \mathcal{M}\left(l_{a_{1}}, \ldots, l_{a_{t}} ; k ; d\right): K_{\lambda_{i}}^{1} \subset M_{j}^{1}, \ldots, K_{\lambda_{i}}^{t} \subset M_{j}^{t}\right\} .
$$


Покрытие

$$
\mathcal{M}\left(l_{a_{1}}, \ldots, l_{a_{t}} ; k ; d\right)=\mathcal{M}_{1} \cup \cdots \cup \mathcal{M}_{\zeta}
$$

обеспечено “двойственным” построением и устройством системы общих представителей.

Некоторое покрытие мы уже произвели, а преобразовать покрытие в разбиение, конечно же, несложно. Более того, имея покрытие (разбиение) совокупности множеств, мы легко приходим и к аналогичному покрытию (разбиению) совокупности векторов

$$
\mathcal{F}\left(l_{a_{1}}, \ldots, l_{a_{t}} ; k ; d\right)=\mathcal{F}_{1} \cup \cdots \cup \mathcal{F}_{\zeta} \quad\left(=\mathcal{F}_{1} \sqcup \cdots \sqcup \mathcal{F}_{\zeta}\right)
$$

Тем не менее, вид оценки (14), которую необходимо обосновать, подсказывает нам, что это покрытие (разбиение) окончательным не является, поскольку пока отсутствуют какие-либо основания предполагать, что диаметры частей $\mathcal{F}_{i}$ меньше $\sqrt{k}$. На следуюшем шаге мы настояшее покрытие измельчим. С этой целью зафиксируем произвольную совокупность $\mathcal{M}_{i}$ и далее будем оперировать именно ею.

Шаг 3. Напомним вид совокупности $\mathcal{M}_{i}$, которую мы желаем покрыть более мелкими частями:

$$
\mathcal{M}_{i}=\left\{\left(M_{j}^{1}, \ldots, M_{j}^{t}\right) \in \mathcal{M}\left(l_{a_{1}}, \ldots, l_{a_{t}} ; k ; d\right): K_{\lambda_{i}}^{1} \subset M_{j}^{1}, \ldots, K_{\lambda_{i}}^{t} \subset M_{j}^{t}\right\}
$$

Как известно, множества $K_{\lambda_{i}}^{1}, \ldots, K_{\lambda_{i}}^{t}$ имеют мошности, равные соответственно некоторым $\eta_{1}, \ldots, \eta_{t}$, причем среди чисел $\eta_{1}, \ldots, \eta_{t}$ есть $t-\theta$ заранее зафиксированных и на данном этапе постоянных величин $p_{\mu_{1}}, \ldots, p_{\mu_{t-\theta}}$; остальные $x_{\nu_{1}}, \ldots$ $\ldots, x_{\nu_{\theta}}$, образующие последовательность из $\widetilde{X}$ и дополняющие упомянутьй выше неизменный набор до исходного (до $\eta_{1}, \ldots, \eta_{t}$ ), могут оказаться в рамках своего определения какими угодно. Далее мы будем считать эти величины произвольными допустимыми и в конечном итоге просто выберем по ним "наихудший вариант" (поскольку мы не можем следить за их реальными значениями).

Заметим, что нашей целью остается такое покрытие (разбиение) совокупности $\mathcal{M}_{i}$, при котором диаметры частей в аналогичном покрытии (разбиении) совокупности $\mathcal{F}_{i}$ становятся меньше, чем $\operatorname{diam} \mathcal{F}\left(l_{a_{1}}, \ldots, l_{a_{t}} ; k ; d\right)=\sqrt{k}$. Однако на величины попарных расстояний между векторами из $\mathcal{F}_{i}$ координаты из множества позиций, лежащих в дизъюнктном объединении $K_{\lambda_{i}}^{1} \sqcup \cdots \sqcup K_{\lambda_{i}}^{t}$, никакого влияния, очевидно, не оказывают: они были зафиксированы, а значит, и вклад их нулевой (ср. доказательство утверждения). В результате необходимо осушествить покрытие даже не самой $\mathcal{M}_{i}$, а совокупности

$$
\widetilde{\mathcal{M}}_{i}=\left\{\left(\widetilde{M}_{j}^{1}, \ldots, \widetilde{M}_{j}^{t}\right): \widetilde{M}_{j}^{\xi}=M_{j}^{\xi} \backslash K_{\lambda_{i}}^{\xi}, \xi=1, \ldots, t,\left(M_{j}^{1}, \ldots, M_{j}^{t}\right) \in \mathcal{M}_{i}\right\}
$$

Понятно, что $\widetilde{\mathcal{M}}_{i}-$ совокупность (упорядоченных) разбиений множества $\Re_{d^{\prime}}$ на части, имеюшие мошности $l_{a_{1}}^{\prime}, \ldots, l_{a_{t}}^{\prime}$, где параметры $d^{\prime}=d-\eta_{1}-\cdots-\eta_{t}$ и $l_{a_{1}}^{\prime}=l_{a_{1}}-\eta_{1}, \ldots, l_{a_{t}}^{\prime}=l_{a_{t}}-\eta_{t}$ известны из формулировки теоремы. Как 
и при доказательстве утверждения на шаге 1 , чтобы упростить запись, освободимся от тех $l_{a_{\xi}}^{\prime}$, которые равны нулю. Окончательно мы придем к необходимости оперировать совокупностью

$$
\overline{\mathcal{M}}_{i}=\left\{\left(\bar{M}_{j}^{1}, \ldots, \bar{M}_{j}^{\tau}\right): \operatorname{card} \bar{M}_{j}^{\xi}=l_{\alpha_{\xi}}^{\prime}>0, \xi=1, \ldots, \tau\right\}
$$

К этой совокупности разбиений множества $\Re_{d^{\prime}}$ применим аналог "двойственной” техники, использованной на предыдущем шаге. Только теперь мы воспользуемся последним классом до сих пор незадействованных параметров: вектором $\mathfrak{m}$, матрицей $\mathfrak{M}$ и числами $D_{5}, L_{5}, S_{5}$. Кроме того, мы прибегнем к помощи теоремы 14.

Рассмотрим совокупность

$$
\overline{\mathcal{K}}=\left\{\left(\bar{K}_{1}^{1}, \ldots, \bar{K}_{1}^{\tau}\right), \ldots,\left(\bar{K}_{D_{5}}^{1}, \ldots, \bar{K}_{D_{5}}^{\tau}\right)\right\} \subset 2^{2^{\Re} d^{\prime}}
$$

состоящую из всех (упорядоченных) разбиений множества $\Re_{d^{\prime}}$ на части $\bar{K}_{j}^{1}, \ldots$ $\ldots, \bar{K}_{j}^{\tau}$, имеющие мощности $\operatorname{card} \bar{K}_{j}^{\xi}=m_{\xi}, j=1, \ldots, D_{5}, \xi=1, \ldots, \tau$. Корректность данного определения вытекает из свойств 1) и 4) параметров m. Аналогично тому, как это было сделано на шаге 2 , отождествим совокупность $\overline{\mathcal{K}}$ с множеством $\Re_{D_{5}}$. В то же время, каждому разбиению $\left(\bar{M}_{j}^{1}, \ldots, \bar{M}_{j}^{\tau}\right)$ из $\overline{\mathcal{M}}_{i}$ мы поставим в соответствие подмножество

$$
\bar{\Lambda}_{j}=\left\{\nu \in \Re_{D_{5}}: \operatorname{card}\left(\bar{K}_{\nu}^{\xi} \cap \bar{M}_{j}^{\mu}\right)=m_{\xi}^{\alpha_{\mu}}, \xi, \mu \in\{1, \ldots, \tau\}\right\} \subset \Re_{D_{5}}
$$

при этом корректность обеспечена свойствами 1)-4) параметров m и $\mathfrak{M}$. Легко проверить, что $\operatorname{card} \bar{\Lambda}_{j}=L_{5}$ для любого $j \in\left\{1, \ldots, \operatorname{card} \overline{\mathcal{M}}_{i}\right\}$ и что $\bar{s}=\operatorname{card} \overline{\mathcal{M}}_{i} \leqslant S_{5}$.

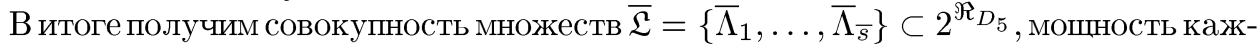
дого элемента которой есть $L_{5}$. Понятно, что, поскольку $\left\{\bar{\lambda}_{1}, \ldots, \bar{\lambda}_{\bar{\zeta}}\right\}, \bar{\zeta}=\zeta(\overline{\mathfrak{L}}),-$ некоторая минимальная с.о.п. для совокупности $\overline{\mathfrak{L}}$, ее мощность не превосходит величины $G\left(D_{5}, L_{5}, \bar{s}\right) \leqslant G\left(D_{5}, L_{5}, S_{5}\right)$ (см. теорему 14$)$.

Произведем, наконец, покрытие $\overline{\mathcal{M}}_{i}$ :

$$
\overline{\mathcal{M}}_{i}=\overline{\mathcal{M}}_{i, 1} \cup \cdots \cup \overline{\mathcal{M}}_{i, \bar{\zeta}}
$$

где

$$
\overline{\mathcal{M}}_{i, j}=\left\{\left(\bar{M}_{\nu}^{1}, \ldots, \bar{M}_{\nu}^{\tau}\right) \in \overline{\mathcal{M}}_{i}: \operatorname{card}\left(\bar{M}_{\nu}^{\xi} \cap \bar{K}_{\bar{\lambda}_{j}}^{\mu}\right)=m_{\mu}^{\alpha_{\xi}}, \xi, \mu \in\{1, \ldots, \tau\}\right\}
$$

которое возможно благодаря двойственной конструкции и свойствам систем общих представителей.

Шаг 4. Теперь суммируем результаты, достигнутые на предыдуших шагах. Во-первых, для каждого $i \in\{1, \ldots, \zeta\}$ мы построили покрытие совокупности $\overline{\mathcal{M}}_{i}$ меньшими совокупностями $\overline{\mathcal{M}}_{i, j}, j=1, \ldots, \bar{\zeta}$. Такое покрытие индуцирует покрытие совокупности $\mathcal{M}_{i}$ некоторыми $\mathcal{M}_{i, j}$, где $i$ и $j$ изменяются в прежних пределах. Во-вторых, такие $\mathcal{M}_{i}, i=1, \ldots, \zeta$, покрывают интересующую нас совокупность 
$\mathcal{M}\left(l_{a_{1}}, \ldots, l_{a_{t}} ; k ; d\right)$. Таким образом, мы получаем покрытие основной совокупности ее частями, количество которых есть $\zeta \bar{\zeta}$. Последняя величина в силу сделанных нами оценок мощностей с. о.п. в различных случаях не превосходит

$$
\left(\sum_{\left(x_{\nu_{1}}, \ldots, x_{\nu_{\theta}}\right) \in \widetilde{\mathcal{X}}}\left(\begin{array}{c}
l_{\varphi_{1}} \\
x_{\nu_{1}}
\end{array}\right) \times \cdots \times\left(\begin{array}{c}
l_{\varphi_{\theta}} \\
x_{\nu_{\theta}}
\end{array}\right)\right) \times\left(\begin{array}{c}
l_{\psi_{1}} \\
p_{\mu_{1}}
\end{array}\right) \times \cdots \times\left(\begin{array}{c}
l_{\psi_{t-\theta}} \\
p_{\mu_{t-\theta}}
\end{array}\right) \times G\left(D_{5}, L_{5}, S_{5}\right) .
$$

Ясно, что, например, параметры $D_{5}, L_{5}, S_{5}$ зависят от многих факторов: от того, как мы фиксировали числа $\theta, \nu_{1}, \ldots, \nu_{\theta}, \mu_{1}, \ldots, \mu_{t-\theta}, p_{\mu_{1}}, \ldots, p_{\mu_{t-\theta}}$, от того, какими оказались числа $x_{\nu_{1}}, \ldots, x_{\nu_{\theta}}$ (ср. с началом шага 3$)$, и от того, наконец, какими выбраны вектор $\mathfrak{m}$ и матрица $\mathfrak{M}$. Следовательно, остается разобраться с кванторами и определить, по каким из перечисленных величин и наборов мы можем взять минимум в оценке на $\zeta \bar{\zeta}$ и по каким из них мы должны в той же оценке произвести максимизацию. Это нетрудно, и мы приходим к искомому неравенству (14).

Итак, мы покрыли совокупность $\mathcal{M}\left(l_{a_{1}}, \ldots, l_{a_{t}} ; k ; d\right)$ должным количеством частей (а значит, и разбили на должное количество частей). Одновременно c $\mathcal{M}\left(l_{a_{1}}, \ldots, l_{a_{t}} ; k ; d\right)$ мы произвели аналогичное покрытие (разбиение) совокупности $\mathcal{F}\left(l_{a_{1}}, \ldots, l_{a_{t}} ; k ; d\right)$. Для завершения доказательства теоремы необходимо проверить, что диаметры полученных частей строго меньше величины $\sqrt{k}$. Однако легко видеть, что это следует из свойства 5) параметров $\mathfrak{m}, \mathfrak{M}, \mathfrak{g}, \mathfrak{G}$. Тем самым теорема 12 полностью доказана.

5.2. Комментарии к доказательству теоремы 13. Мы не проводим для теоремы 13 подробное доказательство, ограничимся лишь тщательным комментарием к нему. На самом деле, этого достаточно: как мы увидим ниже, в доказательстве в значительной мере воспроизводятся аргументы, позволившие нам обосновать теорему 12.

Действительно, доказывая теорему 12, мы, по существу, строили как можно более "экономное" разбиение совокупности векторов $\mathcal{F}\left(l_{a_{1}}, \ldots, l_{a_{t}} ; k ; d\right)$ на части $\mathcal{F}_{i, j}$ меньшего диаметра, находя для этих частей “координатные каркасы" с помощью комбинаторики минимальных систем общих представителей. Для обоснования теоремы 13 нам понадобится осуществить аналогичные построения, а именно, пользуясь свойствами с.о.п., мы покроем совокупность $\mathcal{F}\left(l_{a_{1}}, \ldots, l_{a_{t}}\right.$; $k ; d)$ шарами $\mathcal{B}_{i, j}$ того же диаметра, причем смысл индексов $i, j$ практически полностью сохранится. Только теперь “экономное” разбиение будет достигаться не выбором "каркасов", а выбором центров наших шаров, каковыми последние однозначно определяются, коль скоро ясно, что их радиусы должны не превосходить величины $\frac{\sqrt{k}}{2}$. Заметим, что квадрат числа $\frac{\sqrt{k}}{2}$ есть $\frac{k}{4}$, а значит, становится несколько понятнее принципиальное различие между свойствами 5) параметров $\mathfrak{m}, \mathfrak{M}$ И $\mathfrak{g}, \mathfrak{G}$.

Заявленная схема доказательства теоремы 13 может быть реализована в рамках таких же четырех шагов, как и аналогичная ей схема доказательства теоремы 12 . Более того, первые два шага дословно повторяются, и лишь для удобства мы сократим шаг 2, остановившись (в обозначениях п.5.1) на построении совокупности 
наборов $\left(K_{\lambda_{i}}^{1}, \ldots, K_{\lambda_{i}}^{t}\right), i=1, \ldots, \zeta$, отвечающей минимальной с. о.п. $\left\{\lambda_{1}, \ldots, \lambda_{\zeta}\right\}$ для $\mathfrak{L}$. Совокупности $\mathcal{M}_{i}$ использовать теперь не будем. Третий шаг немного отличается, и мы изложим его подробнее.

Шаг 3. Пусть $d^{\prime}, l_{\alpha_{1}}^{\prime}, \ldots, l_{\alpha_{\tau}}^{\prime}$, а также все прочие параметры (типа $\mathfrak{g}, \mathfrak{G}$ и т. д.) совпадают по своему определению с аналогичными параметрами, возникшими при формулировке теорем 12 и 13 . Для каждого $i \in\{1, \ldots, \zeta\}$ рассмотрим множество

$$
\Re^{i}=\Re_{d} \backslash\left(K_{\lambda_{i}}^{1} \sqcup \cdots \sqcup K_{\lambda_{i}}^{t}\right),
$$

которое, очевидно, можно интерпретировать как $\Re_{d^{\prime}}$ (ср. доказательство теоремы 12). Не забывая о том, что всякое такое множество $\Re_{d^{\prime}}$ имеет свой индекс $i$, мы с целью уменьшения громоздкости изложения этот индекс при записи множества явно указывать не будем. Рассмотрим, далее, совокупность

$$
\overline{\mathcal{M}}_{i}=\left\{\left(\bar{M}_{j}^{1}, \ldots, \bar{M}_{j}^{\tau}\right): \operatorname{card} \bar{M}_{j}^{\xi}=l_{\alpha_{\xi}}^{\prime}, \xi=1, \ldots, \tau\right\}
$$

состоящую из всех допустимых (упорядоченных) разбиений множества $\Re_{d^{\prime}}$ на части, имеющие мощности $l_{\alpha_{\xi}}^{\prime}$ (заметим, что совокупности $\overline{\mathcal{M}}_{i}$ здесь и $\overline{\mathcal{M}}_{i}$ в п. 5.1 немного отличаются друг от друга: последняя вложена в первую, хотя для дальнейшего это не столь важно). Рассмотрим, наконец, другую совокупность разбиений, которая будет иметь и смысл, и обозначение аналогичной совокупности из п. 5.1, но которая, тем не менее, будет от своей предшественницы немного отличаться:

$$
\overline{\mathcal{K}}=\left\{\left(\bar{K}_{1}^{1}, \ldots, \bar{K}_{1}^{\rho}\right), \ldots,\left(\bar{K}_{D_{6}}^{1}, \ldots, \bar{K}_{D_{6}}^{\rho}\right)\right\} \subset 2^{2^{\Re} d^{\prime}}
$$

Она образована всеми (упорядоченными) разбиениями множества $\Re_{d^{\prime}}$ на части $\bar{K}_{j}^{1}, \ldots, \bar{K}_{j}^{\rho}$ мощностей $\operatorname{card} \bar{K}_{j}^{\xi}=g_{\beta_{\xi}}, j=1, \ldots, D_{6}, \xi=1, \ldots, \rho$.

В рамках стандартного двойственного перехода $\overline{\mathcal{K}} \leftrightarrow \Re_{D_{6}},\left(\bar{M}_{j}^{1}, \ldots, \bar{M}_{j}^{\tau}\right) \in$ $\overline{\mathcal{M}}_{i} \leftrightarrow \bar{\Lambda}_{j}$ имеем

$$
\bar{\Lambda}_{j}=\left\{\nu \in \Re_{D_{6}}: \operatorname{card}\left(\bar{K}_{\nu}^{\xi} \cap \bar{M}_{j}^{\mu}\right)=g_{\beta_{\xi}}^{\alpha_{\mu}}, \xi \in\{1, \ldots, \rho\}, \mu \in\{1, \ldots, \tau\}\right\} \subset \Re_{D_{6}}
$$

(см. для проверки корректности этого свойства 1)-4) параметров $\mathfrak{g}$ и $\mathfrak{G})$. Ясно, что $\operatorname{card} \bar{\Lambda}_{j}=L_{6}$ для любого $j \in\left\{1, \ldots, \operatorname{card} \overline{\mathcal{M}}_{i}\right\}$ и что $\operatorname{card} \overline{\mathcal{M}}_{i}=S_{6}$. Для совокупности множеств $\overline{\mathfrak{L}}=\left\{\bar{\Lambda}_{1}, \ldots, \bar{\Lambda}_{S_{6}}\right\} \subset 2^{\Re_{D_{6}}}$, мощность каждого элемента которой есть $L_{6}$, как обычно, находим некоторую минимальную с.о.п. $\left\{\bar{\lambda}_{1}, \ldots, \bar{\lambda}_{\bar{\zeta}}\right\}, \bar{\zeta}=$ $\zeta(\overline{\mathfrak{L}})$, и оцениваем ее мошность, исходя из теоремы $14: \bar{\zeta} \leqslant G\left(D_{6}, L_{6}, S_{6}\right)$. По этой с.о.п., не строя никакого разбиения совокупности $\overline{\mathcal{M}}_{i}$ (ср. шаг 3 из доказательства теоремы 12), мы восстанавливаем совокупность разбиений $\left(\bar{K} \frac{1}{\lambda_{j}}, \ldots, \bar{K} \frac{\rho}{\lambda_{j}}\right)$, $j=1, \ldots, \bar{\zeta}$. На этом новый шаг 3 завершен.

Шаг 4. Новый четвертый шаг так же, как и аналогичный шаг в п. 5.1, является итоговым. В самом деле, мы готовы указать центры $o_{i, j}$ шаров $\mathcal{B}_{i, j}$, покрываюших 
совокупность $\mathcal{F}\left(l_{a_{1}}, \ldots, l_{a_{t}} ; k ; d\right)$. Для этого мы фиксируем $i, j$, а значит, и отвечающие им наборы $\left(K_{\lambda_{i}}^{1}, \ldots, K_{\lambda_{i}}^{t}\right),\left(\bar{K} \bar{\lambda}_{j}, \ldots, \bar{K} \frac{\rho}{\lambda_{j}}\right)$. Понятно, что, каковы бы ни были $i \in\{1, \ldots, \zeta\}$ и $j \in\{1, \ldots, \bar{\zeta}\}$, имеет место разбиение

$$
\Re_{d}=K_{\lambda_{i}}^{1} \sqcup \cdots \sqcup K_{\lambda_{i}}^{t} \sqcup \bar{K} \frac{1}{\lambda_{j}} \sqcup \cdots \sqcup \bar{K} \frac{\rho}{\bar{\lambda}_{j}},
$$

и, значит, корректно задать центры $o_{i, j}=\left(v_{1}, \ldots, v_{d}\right)$, полагая $v_{\nu}=a_{\mu}$, если $\nu \in K_{\lambda_{i}}^{\mu}, \mu=1, \ldots, t$, и $v_{\nu}=\beta_{\mu}$, если $\nu \in \bar{K}_{\bar{\lambda}_{j}}, \mu=1, \ldots, \rho$. Тот факт, что для каждого вектора из $\mathcal{F}\left(l_{a_{1}}, \ldots, l_{a_{t}} ; k ; d\right)$ сушествует содержащий этот вектор шар с центром в некоторой точке $o_{i, j}$, вытекает из свойств систем общих представителей и из свойства 5) параметров, обозначенных готическими буквами. Окончание доказательства (оптимизация) проходит стандартно (см. п. 5.1). Тем самым комментарий к теореме 13 завершен.

Заметим, что, помимо всего прочего, мы прояснили смысл параметров $\beta_{1}, \ldots$ $\ldots, \beta_{\rho}$, оказываюшихся потенциальными величинами координат центров шаров в покрытии. Выбор их в виде полусумм чисел $\alpha_{\nu}$ обусловлен тем, что суммы квадратов разностей их и координат $\alpha_{\nu}$ в векторах из $\mathcal{F}\left(l_{a_{1}}, \ldots, l_{a_{t}} ; k ; d\right)$, представляющие евклидово расстояние, в этом случае достаточно малы, к чему мы и стремимся. Однако не сушествует гарантии того, что выбор каких-нибудь других $\beta_{\mu}$ не приведет к лучшему результату. Именно поэтому мы в п. 3.1 не стали обсуждать вопрос о нахождении точных значений параметров, реализующих минимум в оценках типа (15): найти этот минимум можно, но нельзя утверждать, что именно его следовало искать.

5.3. Несколько иллюстраций к п. 5.1. Чтобы пояснить дополнительно смысл громоздких выкладок, с помощью которых мы доказали теорему 12, а вместе с ней и все ее частные случаи, посмотрим, чем в конкретных ситуациях являются те или иные параметры из п. 5.1. Например, из замечания 4 мы знаем, что в теореме 8 для $(-1,0,1)$-многогранников, на самом деле, $t=3, \theta=2, \nu_{1}=1, \nu_{2}=2$, $\mu_{1}=3$, а $p_{\mu_{1}}=0$. Разумеется, мы вольны были в выборе чисел $\theta$ и других, так что нет ничего удивительного в том, что общую минимизацию, имеющую место в теореме 12 , мы заменили рассмотрением одной из ее конкретных реализаций: почему мы поступили именно так, будет понятно из следующего параграфа. Заметим, что в рамках упомянутой нумерации $a_{1}=1, a_{2}=-1, a_{3}=0$, что, быть может, не вполне коррелирует с последовательностью $-1,0,1$. Действительно, из сочетания замечания 4 и формулировки теоремы 8 следует, что $\varphi_{1}=1, \varphi_{2}=-1$ и $\psi_{1}=0$. Далее, на шаге 2 доказательства появляются величины $D^{*}$ и $L^{*}$. Понятно, что в нынешней ситуации

$$
D^{*}=\sum_{\left(x_{1}, x_{2}\right) \in \mathcal{X}}\left(\begin{array}{c}
d \\
x_{1}
\end{array}\right)\left(\begin{array}{c}
d-x_{1} \\
x_{2}
\end{array}\right), \quad L^{*}=\sum_{\left(x_{1}, x_{2}\right) \in \mathcal{X}}\left(\begin{array}{c}
l_{1} \\
x_{1}
\end{array}\right)\left(\begin{array}{c}
l_{-1} \\
x_{2}
\end{array}\right) .
$$

Наконец, параметры $D_{5}, L_{5}, S_{5}$ естественным образом переходят в параметры $D_{3}^{\prime}$, $L_{3}^{\prime}, S_{3}^{\prime}$. 
Если обратиться к теореме 8 , то станет ясно, что для нее $t=3, \theta=0, \mu_{1}=1$, $\mu_{2}=2, \mu_{3}=3, p_{\mu_{1}}=p_{1}, p_{\mu_{2}}=p_{2}$, а $p_{\mu_{3}}=0$. При этом теперь $\psi_{1}=1, \psi_{2}=-1$ и $\psi_{3}=0$, так что имеем $a_{1}=1, a_{2}=-1, a_{3}=0$. В свою очередь,

$$
D^{*}=\left(\begin{array}{c}
d \\
p_{1}
\end{array}\right)\left(\begin{array}{c}
d-p_{1} \\
p_{2}
\end{array}\right), \quad L^{*}=\left(\begin{array}{c}
l_{1} \\
p_{1}
\end{array}\right)\left(\begin{array}{c}
l_{-1} \\
p_{2}
\end{array}\right),
$$

a $D_{5} \leftrightarrow D_{3}^{\prime \prime}, L_{5} \leftrightarrow L_{3}^{\prime \prime}, S_{5} \leftrightarrow S_{3}^{\prime \prime}$

По-видимому, вполне можно ограничиться рассмотрением этих примеров.

\section{§6. Соотношения между полученными результатами}

В настоящей работе мы получили различные оценки величин $f\left(l_{a_{1}}, \ldots, l_{a_{t}} ; k ; d\right)$ и $g\left(l_{a_{1}}, \ldots, l_{a_{t}} ; k ; d\right)$. Ввиду несомненной громоздкости этих оценок, было бы интересно на конкретных примерах разобраться с тем, как они реально между собой соотносятся. Уже в случае, скажем, неравенств (3) и (4) подобная связь не очевидна. Конечно, эти неравенства были найдены еще в работе [23], и там же было произведено подробное исследование связи между ними. Однако, с одной стороны, то исследование было не полным, а с другой стороны, именно по нему, как по максимально простому из возможных, следует, по-видимому, судить об общем положении дел. Таким образом, сначала рассмотрим оценки (3) и (4).

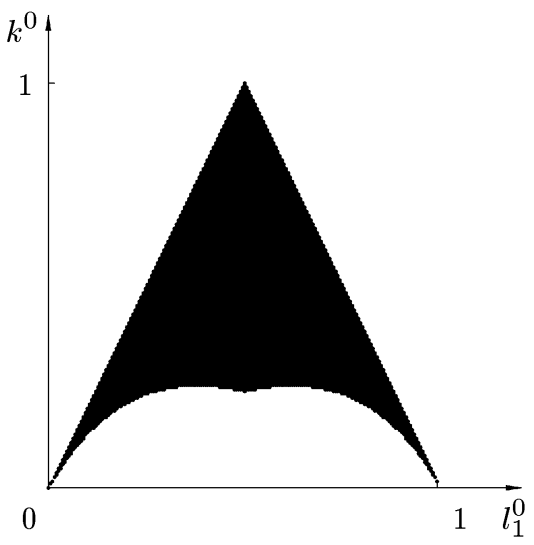

Рис. 1

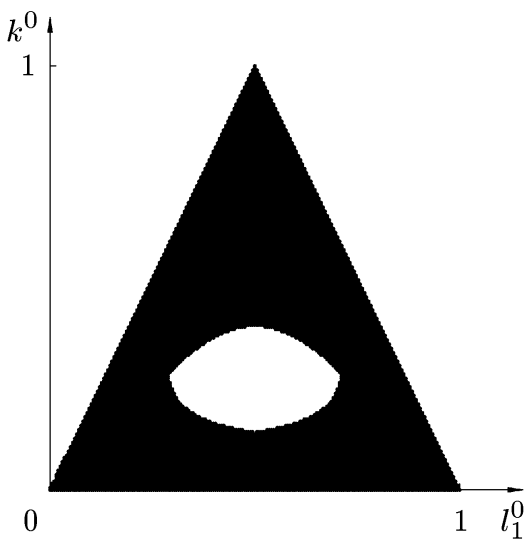

Рис. 2

Оценки (3) и (4) удобно сравнивать графически. В то же время, не следует забывать о том, что мы стремимся так или иначе улучшить общее неравенство Шрамма и Бургейна-Линденштраусса

$$
f(d) \leqslant(d+1) g(d) \leqslant(1.224 \ldots+o(1))^{d},
$$

которому, безусловно, удовлетворяют и наши специальные величины. Поэтому мы представим несколько графиков. Заметим сначала, что наиболее содержательными наши оценки становятся, коль скоро все параметры, от которых они зависят, имеют порядок $d: l_{1} \sim l_{1}^{0} d, l_{0} \sim l_{0}^{0} d, k \sim k^{0} d$, где $l_{1}^{0}, l_{0}^{0}, k^{0} \in(0,1)$ 
(cp. [23]). В такой ситуации нетрудно видеть, что при использовании формулы Стирлинга правые части неравенств (3) и (4) преобразуются в экспоненты вида $\left(\delta_{1}+o(1)\right)^{d}$ и $\left(\delta_{2}+o(1)\right)^{d}$ соответственно $\left(\delta_{1}>1, \delta_{2}>1\right)$. Значит, сравнивать нужно $\delta_{1}, \delta_{2}$ и $1.224 \ldots=\sqrt{\frac{3}{2}}$. На рис. 1 в координатах $l_{1}^{0}$ (ось абсцисс) и $k^{0}$ (ось ординат) изображено множество точек, в которых $\delta_{2}<\delta_{1}$. Симметрия картинки ожидаема (ср. [23]), ограниченность прямыми $k^{0}=2 l_{1}^{0}$ и $k^{0} \leqslant 2-2 l_{1}^{0}$ также ожидаема: в последнем случае достаточно вспомнить определения наших констант. Ясно, что соотношение между $\delta_{1}$ и $\delta_{2}$ весьма нетривиально: нельзя сказать, что одна из величин преобладает над другой слишком часто. Например, если $l_{1}^{0}=k^{0}=0.5$, то $\delta_{1}=\sqrt{2}=1.414 \ldots>\delta_{2}=1.139 \ldots$; если же $l_{1}^{0}=0.3, \quad k^{0}=0.2$, то $\delta_{1}=1.21 \ldots<\delta_{2}=1.33 \ldots$ На рис. 2 в прежних координатах закрашена та область, где $\delta_{1}<1.224 \ldots$ В частности, если $l_{1}^{0}=k^{0}=0.5$, то $\delta_{1}=\sqrt{2}=1.414 \ldots>1.224 \ldots$, а если $l_{1}^{0}=0.3, k^{0}=0.2$, то $\delta_{1}=1.21 \ldots<1.224 \ldots$ Из рис. 3 видно, когда $\delta_{2}<1.224 \ldots$ Скажем, если $l_{1}^{0}=0.3, k^{0}=0.2$, то $\delta_{2}=1.33 \ldots>1.224 \ldots$; если же $l_{1}^{0}=k^{0}=0.5$, то $\delta_{2}=1.139 \ldots<1.224 \ldots$ Наконец, на рис. 4 изображено множество пар $\left(l_{1}^{0}, k^{0}\right)$, для которых $\min \left\{\delta_{1}, \delta_{2}\right\}<1.224 \ldots$ Понятно, почему "дырка" на последней картинке самая маленькая. Ее параметры (“габариты") подробно охарактеризованы в работе [23], она объективно невелика, но при $l_{1}^{0}=0.5, k^{0}=0.3$ все-таки $\min \left\{\delta_{1}, \delta_{2}\right\}=1.31 \ldots>1.224 \ldots$ Заметим, кроме того, что, например, для $l_{1}^{0}=0.2$ и для всех $k^{0} \geqslant 0.3$ вьполнена оценка $\min \left\{\delta_{1}, \delta_{2}\right\}<1.081 \ldots$, которая значительно сильнее общего результата.

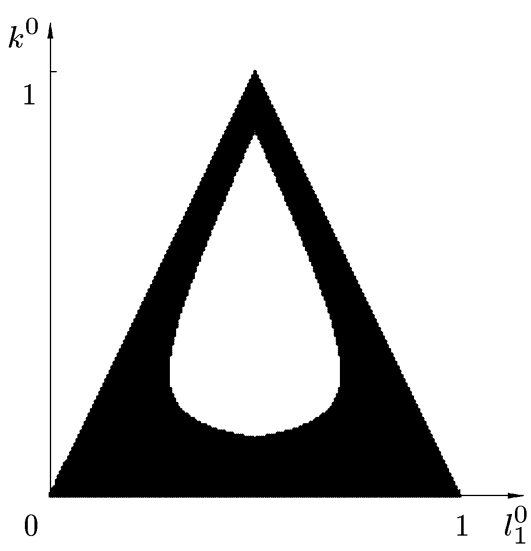

Рис. 3

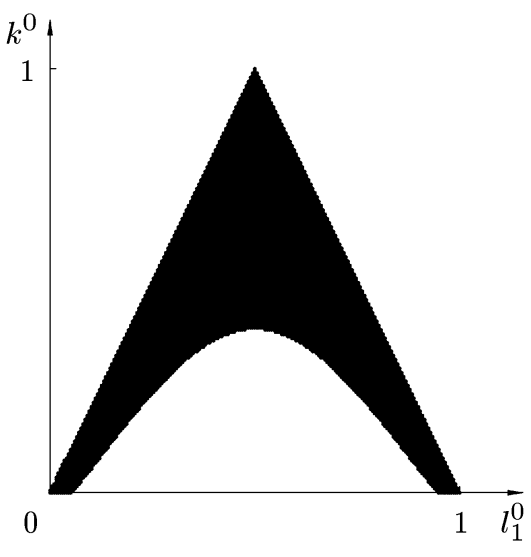

Рис. 4

Далее, в конще п. 5.2 мы обсуждали вопрос о причине выбора чисел $\beta_{i}$ в задаче Грюнбаума в виде полусумм чисел $\alpha_{j}$. Конечно, по сути мы наш выбор прояснили. Тем не менее, интересно привести пример использования нетривиальных полусумм, т.е. величин $\beta_{i}=\frac{\alpha_{j_{1}}+\alpha_{j_{2}}}{2}$ при $j_{1} \neq j_{2}$. Естественно, мы будем искать пример в простейшей ситуации, а именно в $(0,1)$-случае. Пусть в теореме $4 l_{1}=\frac{d}{2}$, $k=\frac{3 d}{4}$. Предположим, что $g_{1 / 2}=0$, т. е. что полусуммы единицы и нуля в качестве возможной координаты центра какого-либо из покрывающих шаров нет. Легко 
убедиться в том, что тогда параметры $\mathfrak{g}, \mathfrak{G}$, минимизирующие оценку (6), имеют следуюший вид: $g_{1}=\frac{d}{2}, g_{1 / 2}=0, g_{0}=\frac{d}{2}, g_{1}^{0}=\frac{3 d}{32}, g_{1}^{1}=\frac{13 d}{32}, g_{1 / 2}^{0}=g_{1 / 2}^{1}=0$, $g_{0}^{1}=\frac{3 d}{32}, g_{0}^{0}=\frac{13 d}{32}$. При этих параметрах в правой части неравенства (6) будет выражение $(1.234 \ldots+o(1))^{d}$. Если взять $g_{1}=\frac{d}{8}, g_{1 / 2}=\frac{3 d}{4} \neq 0, g_{0}=\frac{d}{8}, g_{1}^{0}=0$, $g_{1}^{1}=\frac{d}{8}, g_{1 / 2}^{0}=g_{1 / 2}^{1}=\frac{3 d}{8}, g_{0}^{1}=0, g_{0}^{0}=\frac{d}{8}$, то мы получим оценку

$$
g\left(l_{0}, l_{1} ; k ; d\right) \leqslant\left(2^{1 / 4}+o(1)\right)^{d}=(1.189 \ldots+o(1))^{d}
$$

Пример построен.

В замечании 3 мы утверждали, что оценка (9) лучше, чем оценка (10). Почему это так? Пусть $l_{1}=l_{-1}=\frac{d}{4}, l_{0}=\frac{d}{2}, k=\frac{d}{8}$. Понятно, что $\mathcal{X}$, по сушеству, определяется парами типа $\left(\frac{d}{4}-0, \frac{d}{4}-\frac{d}{16}\right), \ldots,\left(\frac{d}{4}-\frac{d}{32}, \frac{d}{4}-\frac{d}{32}\right), \ldots,\left(\frac{d}{4}-\frac{d}{16}, \frac{d}{4}-0\right)$, хотя ими и не исчерпывается. Поскольку $q_{1}=q_{2}=\frac{d}{4}-\frac{d}{16}$, т.е. $\left(q_{1}, q_{2}\right) \notin \mathcal{X}$, в результате имеем

$$
\sum_{\left(x_{1}, x_{2}\right) \in \mathcal{X}}\left(\begin{array}{c}
l_{1} \\
x_{1}
\end{array}\right)\left(\begin{array}{c}
l_{-1} \\
x_{2}
\end{array}\right) \asymp P(d)\left(\begin{array}{c}
\frac{d}{4} \\
\frac{d}{32}
\end{array}\right)\left(\begin{array}{c}
\frac{d}{4} \\
\frac{d}{32}
\end{array}\right)=(1.207 \ldots+o(1))^{d},
$$

где $P(d)$ - полином. Ясно, что в теореме 8 следует брать $p_{1}=q_{1}=p_{2}=q_{2}$. При этом

$$
\left(\begin{array}{c}
l_{1} \\
p_{1}
\end{array}\right)\left(\begin{array}{c}
l_{-1} \\
p_{2}
\end{array}\right)=(1.45 \ldots+o(1))^{d} .
$$

Наконец, почти очевидно, что за счет выбора тех или иных параметров со штрихами выполнено неравенство

$$
\min _{\mathfrak{m}^{\prime \prime}, \mathfrak{M}^{\prime \prime}} G\left(D_{3}^{\prime \prime}, L_{3}^{\prime \prime}, S_{3}^{\prime \prime}\right) \geqslant \max _{\left(x_{1}, x_{2}\right) \in \mathcal{X}} \min _{\mathfrak{m}^{\prime}, \mathfrak{M}^{\prime}} G\left(D_{3}^{\prime}, L_{3}^{\prime}, S_{3}^{\prime}\right)
$$

Таким образом, преобладание оценки (10) над оценкой (9) в данной ситуации бесспорно.

Неравенства (6) и (7) являются (в проблеме Грюнбаума) прямьми аналогами соответственно неравенств (4) и (3) (в проблеме Борсука). Последние два неравенства мы сравнили достаточно подробно, так что столь детально изучать первые два не имеет смысла. Тем не менее, интересно выяснить, в чем состоит преимущество неравенства (7) над (6). Пусть $l_{1}=l_{1}^{0} d \leqslant \frac{d}{2}, k=k^{0} d$. Тогда нетрудно видеть, что

$$
\begin{gathered}
\min \left\{\left(\begin{array}{c}
l_{1} \\
l_{1}-\frac{k}{2}
\end{array}\right) \min _{\mathfrak{g}^{\prime}, \mathfrak{G}^{\prime}} G\left(D_{2}^{\prime}, L_{2}^{\prime}, S_{2}^{\prime}\right),\left(\begin{array}{c}
l_{0} \\
\frac{k}{2}
\end{array}\right) \min _{\mathfrak{g}^{\prime \prime}, \mathfrak{G}^{\prime \prime}} G\left(D_{2}^{\prime \prime}, L_{2}^{\prime \prime}, S_{2}^{\prime \prime}\right)\right\} \\
=\left(\begin{array}{c}
l_{1} \\
l_{1}-\frac{k}{2}
\end{array}\right) \min _{\mathfrak{g}^{\prime}, \mathfrak{G}^{\prime}} G\left(D_{2}^{\prime}, L_{2}^{\prime}, S_{2}^{\prime}\right) .
\end{gathered}
$$

При этом $\left(\begin{array}{c}l_{1} \\ l_{1}-\frac{k}{2}\end{array}\right)=(1+\delta)^{d}$, где $\delta=\delta\left(l_{1}^{0}, k^{0}\right) \rightarrow 0$ при $k^{0} \rightarrow 0$. В то же время, ввиду выбора параметров со штрихами выполнено равенство

$$
\min _{\mathfrak{g}^{\prime}, \mathfrak{G}^{\prime}} G\left(D_{2}^{\prime}, L_{2}^{\prime}, S_{2}^{\prime}\right)=(1+\gamma)^{d}
$$


где $\gamma$ также стремится к нулю, коль скоро все параметры фиксированы, а $k^{0} \rightarrow 0$. Однако условие 5), наложенное на величины $\mathfrak{m}, \mathfrak{M}, \mathfrak{g}, \mathfrak{G}$, фигурирующие в формулировках соответствующих теорем, показывает, что $\min _{\mathfrak{g}, \mathfrak{G}} G\left(D_{2}, L_{2}, S_{2}\right)$ только увеличивается с уменьшением $k$. Значит, найдется такое $k^{0, *}$, что при всех $k^{0}<k^{0, *}$ оценка (7) лучше оценки (6).

ЗАМЕЧАНИЕ 5 . Каковы бы ни были величины $l_{a_{1}}, \ldots, l_{a_{t}}$, существует такое $k_{0}=k_{0}\left(l_{a_{1}}, \ldots, l_{a_{t}}\right)$, что при всех $k<k_{0}$ вьполнена оценка $\min _{i} q_{i}>0$, влекущая нетривиальность тех результатов, в которых используется $q_{i}$. В частности, и множество $\mathcal{X}$ оказывается нетривиальным. Например, если $l_{1}=l_{-1}=\frac{d}{4}, l_{0}=\frac{d}{2}$, $k=\frac{d}{4}$, то $q_{1}=q_{2}=\frac{d}{8}$, но поскольку $l_{1}=l_{-1}=\frac{d}{4}, l_{0}=\frac{d}{2}, k=\frac{d}{2}$, то $q_{1}=q_{2}=0$.

\section{§7. Приложение: покрытие множеств шарами в произвольной норме}

В предыдущих параграффах мы, в частности, детально изучили "решетчатый" вариант задачи Грюнбаума о покрытии множеств “евклидовыми" шарами. Однако в общей теории покрытий и упаковок (см. [34]-[37]) естественно возникает вопрос о необходимости их реализации в случае, когда, скажем, от покрывающих тел требуется лишь выпуклость и центральная симметричность. Оказывается, технология получения верхних оценок минимального числа "грюнбаумовых" шаров, предложенная выше, применима и к общей ситуации. Сформулируем соответствующий результат.

Пусть $\|\mathbf{x}\|$ - некоторая (произвольная) норма вектора $\mathbf{x}=\left(x_{1}, \ldots, x_{d}\right)$ в пространстве $\mathbb{R}^{d}$, которую мы понимаем теперь как совокупность всех наборов вещественных чисел, имеюших длину $d$. Назовем шаром в этой норме множество

$$
\mathcal{B}_{\|\mathbf{x}\|}(r)=\left\{\mathbf{y}=\left(y_{1}, \ldots, y_{d}\right) \in \mathbb{R}^{d}:\|\mathbf{x}-\mathbf{y}\| \leqslant r\right\}
$$

Ясно, что $\mathbf{x}$ - центр шара, а $r>0$ - его радиус. Тогда (обобщенная) проблема Грюнбаума состоит, очевидно, в нахождении минимального числа $g_{\|\mathbf{x}\|}(d)$ такого, что всякое ограниченное множество $\Omega \subset \mathbb{R}^{d}$ покрьвается $g_{\|\mathbf{x}\|}(d)$ шарами в норме $\|\mathbf{x}\|$. Также и в "решетчатой” задаче: сохраняя все обозначения, нетрудно ввести величину $g_{\|\mathbf{x}\|}\left(l_{a_{1}}, \ldots, l_{a_{t}} ; k ; d\right)$, оценить которую мы, собственно, и стремимся.

Как и прежде, нам необходимо ввести дополнительные параметры. Отметим, что практически все параметры, введенные при формулировке теоремы 13, можно оставить неизменными. Следует внести всего два исправления. Во-первых, в качестве $\beta_{1}, \ldots, \beta_{\rho}$ мы не должны брать исключительно полусуммы чисел $\alpha_{1}, \ldots$ $\ldots, \alpha_{\tau}$; параметры $\beta_{1}, \ldots, \beta_{\rho}$ будут выбираться в зависимости от нормы, в чем и состоит основная сложность. Ниже мы скажем несколько слов об этом, но пока можно считать, что в окончательном неравенстве (которое внешне полностью совпадает с неравенством (15)) есть еще две оптимизации: по $\rho$ и по $\beta_{1}, \ldots, \beta_{\rho}$. Во-вторых, меняется свойство 5) параметров $\mathfrak{g}$ и $\mathfrak{G}$ :

$\left.5^{\prime}\right)$ пусть $\mathbf{x}, \mathbf{y} \in \mathbb{R}^{d^{\prime}}$ таковы, что:

a) $\operatorname{card}\left\{\nu=1, \ldots, d^{\prime}: x_{\nu}=\beta_{i}\right\}=g_{\beta_{i}}, i=1, \ldots, \rho$;

б) $\operatorname{card}\left\{\nu=1, \ldots, d^{\prime}: y_{\nu}=\alpha_{j}\right\}=l_{\alpha_{j}}^{\prime}, j=1, \ldots, \tau$; 
в) $\operatorname{card}\left(\left\{\nu=1, \ldots, d^{\prime}: x_{\nu}=\beta_{i}\right\} \cap\left\{\nu=1, \ldots, d^{\prime}: y_{\nu}=\alpha_{j}\right\}\right)=g_{\beta_{i}}^{\alpha_{j}}$;

пусть, кроме того, $r=r(k)$ подобрано так, чтобы квадрат диаметра шара $\mathcal{B}_{\|\mathbf{x}\|}(r)$ не превосходил $k$; тогда $\|\mathbf{x}-\mathbf{y}\| \leqslant r$, т. е. $\mathbf{y} \in \mathcal{B}_{\|\mathbf{x}\|}(r)$.

Теперь получаем новую теорему, внешне повторяющую теорему 13.

Teорема 15. Пусть $\varphi_{j}=a_{\nu_{j}}, \quad j=1, \ldots, \theta, \quad a \quad \psi_{j}=a_{\mu_{j}}, j=1, \ldots, t-\theta$. Виполнена оченка

$$
\begin{aligned}
& g_{\|\mathbf{x}\|}\left(l_{a_{1}}, \ldots, l_{a_{t}} ; k ; d\right) \leqslant \min _{\theta} \min _{\nu_{1}, \ldots, \nu_{\theta}}\left(\sum_{\left(x_{\nu_{1}}, \ldots, x_{\nu_{\theta}}\right) \in \widetilde{\mathcal{X}}}\left(\begin{array}{c}
l_{\varphi_{1}} \\
x_{\nu_{1}}
\end{array}\right) \times \cdots \times\left(\begin{array}{c}
l_{\varphi_{\theta}} \\
x_{\nu_{\theta}}
\end{array}\right)\right) \\
& \times \max _{\left(x_{\nu_{1}}, \ldots, x_{\nu_{\theta}}\right) \in \widetilde{\mathcal{X}} p_{\mu_{1}}, \ldots, p_{\mu_{t-\theta}}} \min _{\mathfrak{g}, \mathfrak{G}}\left(\begin{array}{c}
l_{\psi_{1}} \\
p_{\mu_{1}}
\end{array}\right) \times \cdots \times\left(\begin{array}{c}
l_{\psi_{t-\theta}} \\
p_{\mu_{t-\theta}}
\end{array}\right) \times G\left(D_{6}, L_{6}, S_{6}\right) .
\end{aligned}
$$

ЗАмечАНИЕ 6. Доказательство теоремы 15 , по сушеству, повторяет доказательство теоремы 13, и мы его здесь не приводим.

ЗАмечание 7. Оптимальный выбор параметров $\rho$ и $\beta_{1}, \ldots, \beta_{\rho}$ в случае произвольной нормы является еще более тонким процессом, чем в случае нормы Евклида. Исследование его представляется трудной задачей, но, например, для классических норм вида

$$
\|\mathbf{x}\|=\left(\left|x_{1}\right|^{\gamma}+\cdots+\left|x_{d}\right|^{\gamma}\right)^{1 / \gamma}, \quad \gamma \geqslant 1,
$$

числа $\beta_{1}, \ldots, \beta_{\rho}$, по-видимому, также стоит выбирать в виде полусумм чисел $\alpha_{\nu}$. Заметим, что для упомянутых норм свойство 5) параметров, обозначенных готическими буквами, снова упрошается:

$$
\left(\sum_{i=1}^{\rho} \sum_{j=1}^{\tau}\left|\beta_{i}-\alpha_{j}\right|^{\gamma} g_{\beta_{i}}^{\alpha_{j}}\right)^{1 / \gamma} \leqslant r=r(k) .
$$

\section{Список литературы}

1. Borsuk K. Drei Sätze über die $n$-dimensionale Euklidische Sphäre // Fundam. Math. 1933. V. 20. P. $177-190$

2. Данцер Л., Грюнбаум Б., Кли В. Теорема Хелли. М.: Мир, 1968.

3. Хадвигер Г., Дебруннер Г. Комбинаторная геометрия плоскости. М.: Наука, 1965.

4. Lenz H. Über die Bedeckung ebener Punktmengen durch solche kleineren Durchmessers // Arch. Math. 1956. V. 4. P. 34-40.

5. Schopp J. Die Abdeckung ebener Bereiche mit konstanten Durchmessern durch drei Kreise von kleinerem Durchmesser // Vortrag, gehalten auf der Geometrie-Tegen in Tihany. Ungarn, 1965.

6. Болтянский В. Г., Гохберг И. Ц. Теоремы и задачи комбинаторной геометрии. М.: Наука, 1965.

7. Boltyanski V.G., Martini H., Soltan P.S. Excursions into combinatorial geometry. Berlin-Heidelberg: Springer-Verlag, 1997. 
8. Hadwiger H. Überdeckung einer Menge durch Mengen kleineren Durchmessers // Comm. Math. Helv. 1945/46. V. 18. P. 73-75; Mitteilung betreffend meine Note: Überdeckung einer Menge durch Mengen kleineren Durchmessers // Comm. Math. Helv. 1946/47. V. 19. P. $72-73$.

9. Kahn J., Kalai G. A counterexample to Borsuk's conjecture // Bulletin (new series) of the AMS. 1993. V. 29. № 1. P. 60-62.

10. Райгородский А. М. Об одной оценке в проблеме Борсука // УМН. 1999. Т. 54. № 2 . C. $185-186$.

11. Rogers C. A. Covering a sphere with spheres // Mathematika. 1963. V. 10. P. 157-164.

12. Schramm O. Illuminating sets of constant width // Mathematika. 1988. V. 35. P. 180-189.

13. Bourgain J., Lindenstrauss J. On covering a set in $\mathbb{R}^{d}$ by balls of the same diameter // Lecture Notes in Math. V. 1469. Berlin: Springer-Verlag, 1991. P. 138-144.

14. Райгородский A.M. Проблема Борсука и хроматические числа метрических пространств // УМН. 2001. Т. 56. №1. С. 107-146.

15. Danzer $L$. On the $k$-th diameter in $E^{d}$ and a problem of Grünbaum // Proc. Colloquium on Convexity. Copenhagen, 1965. P. 41.

16. Schiller F. Zur Berechnung und Abschätzung von Färbungszahlen und der $\vartheta$-Funktion von Graphen: Diplomarbeit. Berlin: TU, 1999.

17. Petersen J. Färbung von Borsuk-Graphen in niedriger Dimension: Diplomarbeit. Berlin: TU, 1998.

18. Payan C. On the chromatic number of cube-like graphs // Discrete Math. 1992. V. 103. P. 271-277.

19. Ziegler G. M. Coloring Hamming graphs, optimal binary codes, and the 0/1-Borsuk problem in low dimensions // Lect. Notes Comput. Sci. 2001. V. 2122. P. 159-171.

20. Ziegler G. M. Lectures on 0/1-polytopes // Polytopes-Combinatorics and Computation / Eds. G. Kalai, G. M. Ziegler. DMV-seminar 29. Basel: Birkhäuser-Verlag, 2000. P. 1-44.

21. Райгородский A.M. Проблема Борсука для $(0,1)$-многогранников и кросс-политопов // ДАН. 2000. Т. 371. № 5. С. 600-603.

22. Райгородский А. М. Проблема Борсука для $(0,1)$-многогранников и кросс-политопов // ДАН. 2002. Т. 384. № 5. С. 593-597.

23. Райгородский A. М. Проблема Борсука для целочисленных многогранников // Матем. сб. 2002. Т. 193. № 10. С. 139-160.

24. Райгородский A. М. Проблемы Борсука, Грюнбаума и Хадвигера для некоторых классов многогранников и графов // ДАН. 2003. Т. 388. №6. С. 738-742.

25. Raigorodskii A. M. The Borsuk partition problem // Lect. Note Ser. of the London Math. Soc. 2005.

26. Райгородский A. M. Об одной задаче оптимального покрытия множеств шарами // Чебышевский сборник. 2002. Т. 3. №2(4). С. 100-106.

27. Linial N., Meshulam R., Tarsi M. Matroidal bijections between graphs // J. Combin. Theory. Ser. B. 1988. V. 45. № 1. P. 31-44.

28. Райгородский A.M. Системы общих представителей // Фунд. и прикл. математика. 1999. T. 5. № 3. C. 851-860.

29. Кузюрин $H . H$. Асимптотическое исследование задачи о покрытии // Проблемы кибернетики. 1980. № 37 . С. 19-56.

30. Turán P. Egy grafelmeletiszelsoertek feladatrol // Mat. Fiz. Lapok. 1941. V. 48. P. $436-452$.

31. Katona G., Nemetz T., Simonovitz M. On a graph problem of Turan // Mat. Lapok (in Hungarian). 1964. V. 15. P. 228-238.

32. Füredi Z. Turán's type problems. Surveys in Combinatorics // Proc. of the 13th British Combin. Conference / Ed. A.D. Keedwell. Cambridge: Cambridge Univ. Press, 1991. P. 253-300. 
33. Райгородский $A . M$. Дефект допустимых шаров и октаэдров в решетке и системы общих представителей // Матем. сб. 1998. Т. 189. №6. С. 117-141.

34. Касселс Джс. Введение в геометрию чисел. М.: Мир, 1965.

35. Gruber P. M., Lekkerkerker C. G. Geometry of numbers. Amsterdam: North-Holland, 1987.

36. Конвей Дж., Слоэн Н. Упаковки шаров, решетки и группы. М.: Мир, 1990.

37. Роджерс К. Укладки и покрытия. М.: Мир, 1968.

Московский государственный университет им. М. В. Ломоносова

Поступило в редакцию

E-mail: raigor@avangard.ru

01.10.2003 